Renormalization for the boundary of chaos in piecewise monotonic maps with a single discontinuity

Glendinning, Paul

2008

MIMS EPrint: 2008.91

Manchester Institute for Mathematical Sciences

School of Mathematics

The University of Manchester

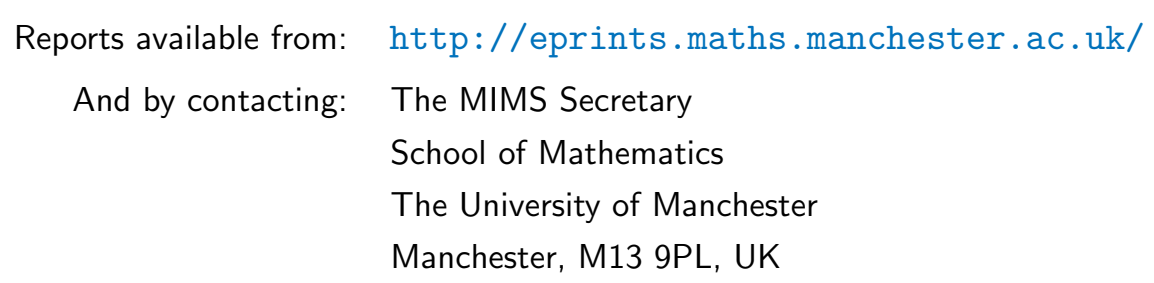

ISSN 1749-9097 


\title{
RENORMALIZATION FOR THE BOUNDARY OF CHAOS IN PIECEWISE MONOTONIC MAPS WITH A SINGLE DISCONTINUITY
}

\author{
PAUL GLENDINNING \\ SCHOOL OF MATHEMATICS \\ AND \\ CICADA \\ (CENTRE FOR INTERDISCIPLINARY COMPUTATIONAL AND DYNAMICAL ANALYSIS) \\ UNIVERSITY OF MANCHESTER \\ OXFORD ROAD, MANCHESTER M13 9PL \\ UNITED KINGDOM. \\ E-MAIL: P.A.GLENDINNING@MANCHESTER. AC.UK
}

\begin{abstract}
Monotonic maps with a single discontinuity arise in a variety of situations. We describe the infinite sets of periods for such maps on the boundary of chaos; this gives a sense of the routes to chaos in such maps. The description involves an explicit subshift of finite type which describes the sequences of different renormalizations possible in these maps.
\end{abstract}

Keywords: renormalization, piecewise monotonic map, homoclinic bifurcation, hybrid system

\section{Introduction}

One-dimensional piecewise monotonic maps with a single discontinuity arise naturally in many situations. Early work was mostly associated with the study of global bifurcations, and in this case the maps have slopes which (typically) tend to zero or infinity as the point of discontinuity is approached from above or below $[12,13,14,15,17]$. On the other hand, linear maps are good models in a range of applications involving switching, so they have been studied within the literature of hybrid systems $[4,5,6,7]$. A number of recent works have looked at these linear models in more detail $[1,2,3]$.

This paper is concerned with routes to chaos in these maps. One of the most important steps towards understanding the possible routes to chaos involves determining the structure of maps on the boundary of chaos (in the sense of positive topological

Date: October 24, 2008. 

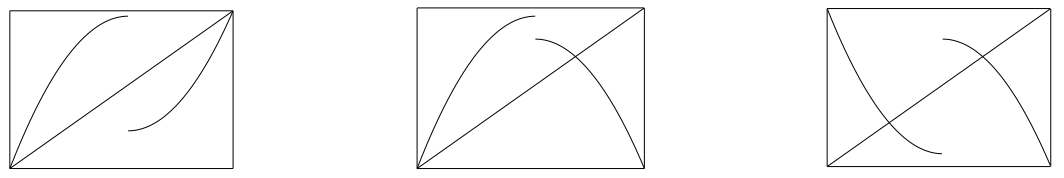

Figure 1. Monotonic maps with a single discontinuity: from left to right Class A (orientable), Class B (semi-orientable), and Class C (nonorientable). Maps in class D are obtained from maps in class B by the transformation $x \rightarrow-x$. In each case the interval is $\left[z_{0}, z_{1}\right]$, with the definitions given in the text.

entropy). In $[13,14]$ a new route to chaos for a class of differential equations modelled by semi-flows on Lorenz surfaces was described. Return maps for these flows are piecewise monotonic with a single discontinuity such as the maps shown in Figure 1b,c. To obtain this new (anharmonic) route to chaos various restrictions must be put on the classes of maps considered. One of the aims of this paper is to place the anharmonic route to chaos in the more general context of transitions to chaos in maps like those shown in Figure 1. We do this by concentrating on maps on the boundary of chaos which are infinitely renormalizable, characterizing these maps by the set of periods which exist for each map. More precisely, let $f: I \rightarrow I$ be a map of the interval $I$, then the set of (minimal) periods of $f, P(f)$, is defined to be

$$
P(f)=\left\{n \in \mathbb{N} \mid f^{n}(x)=x \text { some } x \in I \text {, and } f^{i}(x) \neq x, 1 \leq i<n\right\}
$$

Thus $P(f)$ is a (finite or infinite) set of positive integers. In the sense that families of maps which pass through a given map $f$ on the boundary of chaos must create orbits with periods in $P(f)$ before becoming chaotic, this characterization gives some insight into the bifurcations which must occur in transitions to chaos via the map $f$. If a map is on the boundary of chaos and is not infinitely renormalizable it will still be renormalizable a finite number of times (possibly zero) and in this sense this paper gives a complete description of the possible periods on the boundary of chaos.

The maps in Figure 1 have three properties in common, there is a discontinuity at $x=0$, they are continuous and monotonic in $x<0$ and in $x>0$, and there are continuously differentiable $(x \neq 0)$ with slope tending to zero as $x$ tends to zero from 
either above or below. Although this latter property is not fundamental here it is natural in the context of global bifurcation theory [15]. The boundary of chaos in the case that both branches of the map are increasing (called Class A [15], Fig. 1(a)) has been described by Tresser [19]. Essentially the boundary of chaos (in a natural parameter space) consists of points on which maps have an infinite set of periods $\left(p_{n}\right)$ related by $p_{n+1}=a_{n} p_{n}, a_{n}>1$, and line segments on which maps have a finite set of periods (also satisfying $p_{n+1}=a_{n} p_{n}$ ). Since there are a number of papers on these cases, no further details will be given here $[11,17,19]$.

The remaining three classes of maps are class $\mathrm{B}$ (increasing in $x<0$, decreasing in $x>0$ ), class $\mathrm{C}$ (decreasing in both $x<0$ and $x>0$ ) and class $\mathrm{D}$ (decreasing in $x<0$ and increasing in $x>0$ ). Note that maps in class B and class D are equivalent under the transformation $x \rightarrow-x$. For all these cases there are regions (in a suitably chosen parameter space) where maps on the boundary of chaos have the infinite set of periods $\left(p_{n}\right)$ with

$$
p_{n+1}=2 p_{n}+(-1)^{n}
$$

This corresponds to transitions to chaos via the anharmonic route described in [13, 14]. By making explicit some remarks in $[8,15]$ it is possible to set up a subshift of finite type which describes how the boundary of chaos is mapped to itself under a number of renormalization operations. The derivation of this subshift is the main result here: it also enables us to give (implicitly) a description of all the possible infinite sets of periods for maps on the boundary of chaos in classes B, C and D.

The methods used here echo the standard arguments for unimodal maps. For a family of unimodal maps, either the dynamics is simple (there is a stable fixed point), or an induced map can be defined by looking at the second iterate of the map restricted to an appropriate interval. If this does not map this interval into itself then the map is chaotic (in the sense of having positive topological entropy), and if it does the process can repeat. The only difference here is that the number of zero entropy renormalizations which can occur is bigger than one, and the different zero entropy renormalizations form a subshift of finite type themselves, however, the principle remains the same.

\section{Some Definitions: MAPS AND PARAMETER SPACE}

We begin with the basic definition of the four classes of maps being considered and then go on to describe the parametrization of families of maps in each class. In all cases the interval the interval $I=\left[z_{0}, z_{1}\right]$ with $z_{0}<c<z_{1}$ and the point of discontinuity of the map is at $x=c$.

Definition 2.1: A map $f:\left[z_{0}, z_{1}\right] \rightarrow\left[z_{0}, z_{1}\right]$ is monotonic with a single discontinuity (msdc) if

(i) $f$ is continuous on $\left[z_{0}, z_{1}\right] \backslash\{c\}$; 
(ii) $\left.f\right|_{\left(z_{0}, c\right)}$ and $\left.f\right|_{\left(c, z_{1}\right)}$ is strictly monotonic; and

(iii) $f\left(z_{i}\right) \in\left\{z_{0}, z_{1}\right\}, i=0,1$.

Note that the third condition is easy to relax, but we retain it as the boundary points will be important in the renormalization process. The four different classes of maps are obtained by choosing the branches of $f$ to be increasing or decreasing in different combinations, together with simple conditions at the end-points of the interval. We use the notation $f\left(c^{-}\right)=\lim _{x \uparrow c} f(x)$ and $f\left(c^{+}\right)=\lim _{x \downarrow c} f(x)$.

Definition 2.2: A map $f:\left[z_{0}, z_{1}\right] \rightarrow\left[z_{0}, z_{1}\right]$ is in class $\mathrm{A}$ if it is msdc and

(i) $\left.f\right|_{\left(z_{0}, c\right)}$ and $\left.f\right|_{\left(c, z_{1}\right)}$ are strictly increasing; and

(ii) $f\left(z_{0}\right)=z_{0}$ and $f\left(z_{1}\right)=z_{1}$.

As noted in the introduction, the boundary of chaos of maps in class $\mathrm{A}$ is described in $[11,19]$.

Definition 2.3: A map $f:\left[z_{0}, z_{1}\right] \rightarrow\left[z_{0}, z_{1}\right]$ is in class B if it is msdc and

(i) $\left.f\right|_{\left(z_{0}, c\right)}$ is strictly increasing and $\left.f\right|_{\left(c, z_{1}\right)}$ is strictly decreasing; and

(ii) $f\left(z_{0}\right)=f\left(z_{1}\right)=z_{0}$.

Definition 2.4: A map $f:\left[z_{0}, z_{1}\right] \rightarrow\left[z_{0}, z_{1}\right]$ is in class $\mathrm{C}$ if it is msdc and

(i) $\left.f\right|_{\left(z_{0}, c\right)}$ and $\left.f\right|_{\left(c, z_{1}\right)}$ are strictly decreasing;

(ii) $f\left(z_{i}\right)=f\left(z_{1-i}\right),=0,1$.

Definition 2.5: A map $f:\left[z_{0}, z_{1}\right] \rightarrow\left[z_{0}, z_{1}\right]$ is in class $\mathrm{D}$ if it the map $g$ : $\left[-z_{1},-z_{0}\right] \rightarrow\left[-z_{1},-z_{0}\right]$ defined by $g(x)=-f(-x)$, is in class B.

Maps in these four classes are determined (up to a semi-conjugacy which collapses only sinks and their preimages and wandering intervals to points) by two topological invariants: the kneading sequences of $c^{+}$and $c^{-}[8]$. Hence it seems natural to work in a two-parameter space. All the results below are strictly true in the space of kneading invariants, but to make matters more approachable we assume that there exist full two-parameter families of maps in each class so that the parameter space can be taken to be a connected closed subset of $\mathbb{R}^{2}$, cf. [15]. For example, the maps

$$
A_{\mu, \nu}(x)= \begin{cases}\nu-x^{2} & \text { if } x<0 \\ -\mu+x^{2} & \text { if } x>0\end{cases}
$$

is in class A with $c=0$ provided $(\mu, \nu) \in D_{A}$ defined by

$$
D_{A}=\left\{(\mu, \nu) \mid-\frac{1}{4} \leq \nu \leq \mu(\mu+1) \text { and }-\frac{1}{4} \leq \mu \leq \nu(\nu+1)\right\}
$$

see Figure 2a. We shall refer to the region $D_{A}$ as an A-box. Ideally we would work with two-parameter full families of maps, i.e. families which realise every possible 

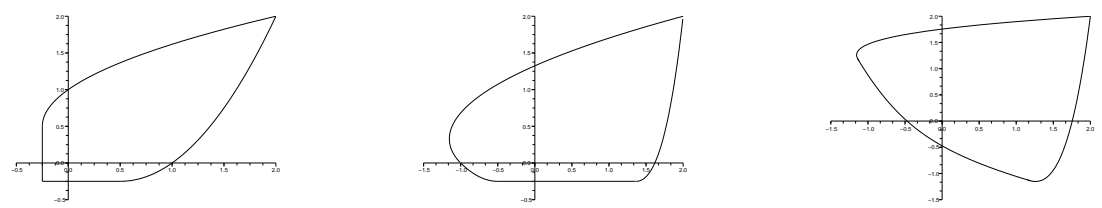

Figure 2. Parameter boxes for Class A (orientable), Class B (semiorientable), and Class C (non-orientable).

kneading invariant of the class, and more generally, an A-box for a full family of maps in class A parameterised by $\mu$ and $\nu$, is a region bounded by the curves $U_{1}$ to $U_{4}$ defined by

$$
\begin{aligned}
& U_{1}=\left\{\mathbf{a} \in \mathbb{R}^{2} \mid f_{\mathbf{a}}^{\prime}\left(z_{0}\right)=1\right\} \\
& U_{2}=\left\{\mathbf{a} \in \mathbb{R}^{2} \mid f_{\mathbf{a}}^{\prime}\left(z_{1}\right)=1\right\} \\
& U_{3}=\left\{\mathbf{a} \in \mathbb{R}^{2} \mid f_{\mathbf{a}}\left(c^{-}\right)=z_{1}\right\} \\
& U_{4}=\left\{\mathbf{a} \in \mathbb{R}^{2} \mid f_{\mathbf{a}}\left(c^{+}\right)=z_{0}\right\}
\end{aligned}
$$

for which every kneading invariant is realised for at least one choice of a. Note that the first two are the loci of saddlenode bifurcations creating the boundary fixed points, and the second to are homoclinic bifurcation points beyond which the interval $\left[z_{0}, z_{1}\right]$ is no longer invariant. For simplicity, we assume that these conditions do indeed define segments of continuous curves in the plane and that they bound a closed, connected set, $D_{A}$. Note that the quadratic family $A_{\mu, \nu}$ is not full $[8,9,12]$.

Similar definitions can be given in the cases of full families of maps in class B (and hence class D) and class $\mathrm{C}$ (see Figure $2 \mathrm{~b}, \mathrm{c})$. For a full family of maps, $\left(f_{\mathbf{a}}\right)$ in class $\mathrm{B}$, with $\mathbf{a} \in \mathbb{R}^{2}$, define

$$
\left.\begin{array}{l}
V_{1}=\left\{\mathbf{a} \in \mathbb{R}^{2} \mid f_{\mathbf{a}}^{\prime}\left(z_{0}\right)=1\right\} \\
V_{2}=\left\{\mathbf{a} \in \mathbb{R}^{2} \mid f_{\mathbf{a}}\left(c^{-}\right)=z_{1}\right\} \\
V_{3}=\left\{\mathbf{a} \in \mathbb{R}^{2} \mid f_{\mathbf{a}}\left(c^{+}\right)=z_{0}\right.
\end{array}\right\}
$$

Then a B-box is the region bounded by these three curves. Note that both $V_{1} \cap V_{2}$ and $V_{1} \cap V_{3}$ correspond to degenerate cases of the maps for which we allow $z_{1}=c$. In terms of the simple quadratic family

$$
B_{\mu, \nu}(x)= \begin{cases}\nu-x^{2} & \text { if } x<0 \\ \mu-x^{2} & \text { if } x>0\end{cases}
$$


these curves are given by

$$
\begin{aligned}
& V_{1}=\left\{(\mu, \nu) \mid \nu=-\frac{1}{4}\right\} \\
& V_{2}=\left\{(\mu, \nu) \mid \nu=\left(\mu-\nu^{2}\right)+\left(\mu-\nu^{2}\right)^{2}\right\} \\
& V_{3}=\left\{(\mu, \nu) \mid \nu=\left(\mu-\mu^{2}\right)+\left(\mu-\mu^{2}\right)^{2}\right\}
\end{aligned}
$$

Finally, for full families $\left(g_{\mathbf{a}}\right)$ in class $\mathrm{C}$, with a $\mathbf{a} \in \mathbb{R}^{2}$, define

$$
\left.\begin{array}{l}
W_{1}=\left\{\mathbf{a} \in \mathbb{R}^{2} \mid\left(g_{\mathbf{a}}^{2}\right)^{\prime}\left(z_{i}\right)=1\right\} \\
W_{2}=\left\{\mathbf{a} \in \mathbb{R}^{2} \mid \begin{array}{l}
g_{\mathbf{a}}\left(c^{+}\right)=z_{1} \\
W_{3}
\end{array}\right\}\left\{\mathbf{a} \in \mathbb{R}^{2} \mid g_{\mathbf{a}}\left(c^{-}\right)=z_{0}\right.
\end{array}\right\}
$$

Then these three curves enclose the region $D_{C}$. As with case $\mathrm{B}$, the intersections of these curves correspond to degenerate cases. Again, these curves are easy to calculate for the quadratic family

$$
C_{\mu, \nu}(x)= \begin{cases}-\nu+x^{2} & \text { if } x<0 \\ \mu-x^{2} & \text { if } x>0\end{cases}
$$

$W_{1}$ does not have a simple close form (though it can be given explicitly using solutions of cubic equations), and is shown numerically in Figure 2c, and

$$
\begin{aligned}
& W_{2}=\left\{(\mu, \nu) \mid \mu+\nu=\left(\mu-\mu^{2}\right)^{2}\right\} \\
& W_{3}=\left\{(\mu, \nu) \mid \mu+\nu=\left(\nu-\nu^{2}\right)^{2}\right\}
\end{aligned}
$$

\section{KneAding Theory}

Kneading theory provides a symbolic description of iterates of maps. Maps can be identified (up to sets of periodic orbits with the same code and homtervals, i.e. intervals on which $f^{n}$ is monotonic for all $n$; these intervals can include parts of the local basins of attraction of stable periodic orbits if they exist) by their kneading invariant: a pair of sequences associated with orbits obtained by approaching the critical point $c$ from above or below. The material presented here is standard, although the different cases are not usually described together.

Let $f: I \rightarrow I$ be a msdc map with discontinuity $c \in I$, and define

$$
a(x)=\left\{\begin{array}{rr}
-1 & \text { if } x<c \\
+1 & \text { if } x>c
\end{array}\right.
$$

If $x$ is not a preimage of $c$ then define the itinerary of $x, I(x)$ to be the sequence $I(x)=a_{0} a_{1} a_{2} a_{3} \ldots$ where $a_{i}=a\left(f^{i}(x)\right), i=0,1,2,3, \ldots$ To extend this definition to pre-images of $c$ define a metric $d$ on $\{-1,+1\}^{\mathbb{N}_{0}}$ by

$$
d(\mathbf{a}, \mathbf{b})=\sum_{n=0}^{\infty} \frac{\left|a_{n}-b_{n}\right|}{2^{n+1}}
$$


If $f^{n}(x)=c$ for some $x \in I$ then define the upper itinerary, $I\left(x_{+}\right)$and the lower itinerary, $I\left(x_{-}\right)$by

$$
I\left(x_{+}\right)=\lim _{y \downarrow x} I(y), \quad I\left(x_{-}\right)=\lim _{y \uparrow x} I(y)
$$

where the limits are taken through points which are not preimages of $c$. Clearly upper and lower itineraries can also be defined for points which are not preimages of $c$, but the $I\left(x_{-}\right)=I\left(x_{+}\right)=I(x)$.

The pair consisting of the upper and lower itinerary of the point of discontinuity itself is called the kneading invariant of $f, k(f)$. in other words, if $k_{+}(f)=I\left(c_{+}\right)$ and $k_{-}(f)=I\left(c_{-}\right)$then

$$
k(f)=\left(k_{+}(f), k_{-}(f)\right)
$$

When the map $f$ is clear from the context, the argument $f$ will be dropped.

The order of points on the real line with different itineraries can be deduced by looking at the itineraries of the points. For this we need to define four order on sequences in $\{-1,+1\}^{\mathbb{N}_{0}}$, which will be relevant to the four classes of msdc maps defined earlier. Here and blow we use the notation $\mathbb{N}_{0}=\{0,1,2, \ldots\}$ to remind the reader that indexing typically begins with the subscript zero. For sequences $\mathbf{a}$ and $\mathbf{b}$ in $\{-1,+1\}^{\mathbb{N}_{0}}$ and maps in class A (orientable) define $\prec_{o}$ by

$$
\mathbf{a} \prec_{o} \mathbf{b}
$$

if and only if either $a_{0}<b_{0}$ or $a_{i}=b_{i}, 0 \leq i \leq n-1$ and $a_{n}<b_{n}$. This is a complete order on $\{-1,+1\}^{\mathbb{N}_{0}}$ and is often called the lexicographical order.

For sequences $\mathbf{a}$ and $\mathbf{b}$ in $\{-1,+1\}^{\mathbb{N}_{0}}$ and maps in class $\mathrm{B}$ (semi-orientable) define $\prec_{\text {so+ }}$ by

$$
\mathbf{a} \prec_{\text {so+ }} \mathbf{b}
$$

if and only if either $a_{0}<b_{0}$ or $a_{i}=b_{i}, 0 \leq i \leq n-1$ and

$$
a_{n} \Pi_{0}^{n-1}\left(-a_{k}\right)<b_{n} \Pi_{0}^{n-1}\left(-b_{k}\right)
$$

so if the number of $+1 \mathrm{~s}$ in $a_{0} a_{1} \ldots a_{n-1}$ is odd the usual order is reversed. This is a complete order on $\{-1,+1\}^{\mathbb{N}_{0}}$ and is sometimes called the unimodal order.

For sequences $\mathbf{a}$ and $\mathbf{b}$ in $\{-1,+1\}^{\mathbb{N}_{0}}$ and maps in class D (semi-orientable) define $\prec_{\text {so- }}$ by

$$
\mathbf{a} \prec_{s o-} \mathbf{b}
$$

if and only if either $a_{0}<b_{0}$ or $a_{i}=b_{i}, 0 \leq i \leq n-1$ and

$$
a_{n} \Pi_{0}^{n-1}\left(a_{k}\right)<b_{n} \Pi_{0}^{n-1}\left(b_{k}\right)
$$

so if the number of $-1 \mathrm{~s}$ in $a_{1} \ldots a_{n-1}$ is odd the usual order is reversed. This is a complete order on $\{-1,+1\}^{\mathbb{N}_{0}}$ and is also a unimodal order.

For sequences $\mathbf{a}$ and $\mathbf{b}$ in $\{-1,+1\}^{\mathbb{N}_{0}}$ and maps in class D (non-orientable) define $\prec_{n o}$ by

$$
\mathbf{a} \prec_{n o} \mathbf{b}
$$


if and only if either $a_{0}<b_{0}$ or $a_{i}=b_{i}, 0 \leq i \leq n-1$ and

$$
(-1)^{n} a_{n}<(-1)^{n} b_{n}
$$

Again, this is a complete order on $\{-1,+1\}^{\mathbb{N}_{0}}$.

Lemma 1. Let $f: I \rightarrow I$ be a msdc map and $\prec_{\alpha}$ the appropriate order. If $I\left(x_{+}\right) \prec_{\alpha}$ $I\left(y_{-}\right)$then $x<y$.

This is a standard result and we omit the proof here. The action of the map on points induces a map on itineraries: the shift map, $\sigma$, defined by $\sigma\left(a_{0} a_{1} a_{2} a_{3} \ldots\right)=$ $a_{1} a_{2} a_{3} a_{4} \ldots$ The relationship between $\sigma$ and $f$ is given by the following lemma (which is again standard).

Lemma 2. Let $f: I \rightarrow I$ be a msdc map and $x \neq c$. Then

$$
I\left(f(x)_{ \pm}\right)=\sigma I\left(x_{ \pm}\right)
$$

if $f$ is increasing at $x$, and

$$
I\left(f(x)_{ \pm}\right)=\sigma I\left(x_{\mp}\right)
$$

if $f$ is decreasing at $x$.

We now move on to consider which sequences can be kneading sequences and to what extent the itineraries of points are determined by the kneading sequence. Before we do this, the very simple cases need to be treated separately.

\section{THE SIMPLE CASES}

This section is exceptionally dull. The reader is strongly advised to look at the pictures and ignore the statements. However, the cases here are important and so cannot be omitted.

Lemma 3. (The orientable case - class A) Let $f: I \rightarrow I$ be in class $A$ and let $X_{+}$ (resp. $X_{-}$) denote the fixed points of $f$ in $x \geq c$ (resp. $\left.x \leq c\right)$.

(i) If $f\left(c_{-}\right) \leq c$ and $f\left(c_{+}\right) \geq c$ then

(1) $\left(k_{+}, k_{-}\right)=\left((+1)^{\infty},(-1)^{\infty}\right)$;

(2) $I(x)=(-1)^{\infty}$ for all $x<c$ and $I(x)=(+1)^{\infty}$ for all $x>c$;

(3) $\Omega(f)=X_{-} \cup X_{+}$.

(ii) If $f\left(c_{-}\right) \leq c$ and $f\left(c_{+}\right)<c$ then

(1) $\left(k_{+}, k_{-}\right)=\left((+1)(-1)^{\infty},(-1)^{\infty}\right)$;

(2) $I(x)=(-1)^{\infty}$ for all $x<c$ and if $x>c$ then either $I(x)=(+1)^{n}(-1)^{\infty}$ for some $n>0$ or $I(x)=(+1)^{\infty}$ or $I\left(x_{-}\right)=(+1)^{n}(-1)^{\infty}$ and $I\left(x_{+}\right)=$ $(+1)^{n+1}(-1)^{\infty}$

(3) $\Omega(f)=X_{-} \cup X_{+}$.

(iii) If $f\left(c_{-}\right)>c$ and $f\left(c_{+}\right) \leq c$ then the situation is as in case (ii) above, but with the roles of $x<c$ and $x>c$ reversed. 

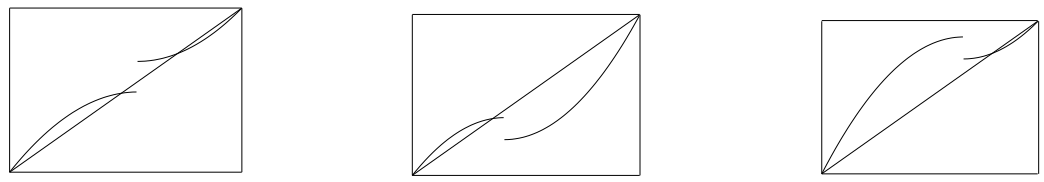

Figure 3. Maps in the three easy cases of Lemma 3.

The situation is as shown in Figure 4; proofs come from standard results for monotonic maps.

Lemma 4. (The semi-orientable case - class B; increasing in $x<c$ and decreasing in $x>c)$ Let $f: I \rightarrow I$ be in class $B$ and let $X_{-}$denote the fixed points of $f$ in $x \leq c$ and $X_{+}^{1,2}$ denote the fixed points and points of period two (possibly empty) in $x \geq c$. (i) If $f\left(c_{-}\right) \leq c$ and $f\left(c_{+}\right) \leq c$ then

(1) $\left(k_{+}, k_{-}\right)=\left((+1)(-1)^{\infty},(-1)^{\infty}\right)$;

(2) $I(x)=(-1)^{\infty}$ for all $x<c$ and $I(x)=(+1)(-1)^{\infty}$ for all $x>c$;

(3) $\Omega(f)=X_{-}$.

(ii) If $f\left(c_{-}\right) \leq c, f\left(c_{+}\right)>c$ and $f^{2}\left(c_{+}\right) \geq c$ then

(1) $\left(k_{+}, k_{-}\right)=\left((+1)^{\infty},(-1)^{\infty}\right)$;

(2) $I(x)=(-1)^{\infty}$ for all $x<c$ and if $x>c$ then either $I(x)=(+1)(-1)^{\infty}$ or $I(x)=(+1)^{\infty}$ or $I\left(x_{-}\right)=(+1)^{\infty}$ and $I\left(x_{+}\right)=(+1)(-1)^{\infty}$;

(3) $\Omega(f)=X_{-} \cup X_{+}^{1,2}$.

(iii) If $f\left(c_{-}\right) \leq c, f\left(c_{+}\right)>c$ and $f^{2}\left(c_{+}\right)<c$ then

(1) $\left(k_{+}, k_{-}\right)=\left((+1)^{2}(-1)^{\infty},(-1)^{\infty}\right)$;

(2) $I(x)=(-1)^{\infty}$ for all $x<c$ and if $x>c$ then either $I(x)=(+1)^{\infty} I(x)=$ $(+1)^{n}(-1)^{\infty}$ for some $n>0$ or $I\left(x_{-}\right)=(+1)^{k}(-1)^{\infty}$ and $I\left(x_{+}\right)=(+1)^{k+2}(-1)^{\infty}$ for some $k>1$;

(3) $\Omega(f)=X_{-} \cup X_{+}^{1,2}$.

Again, the different possibilities are illustrated in Figure 4, and the situation for maps in class $\mathrm{D}$ is the same as for class $\mathrm{B}$ but with the roles of $x>c$ and $x<c$ reversed and hence the roles of the symbols \pm 1 are also reversed. Finally we have the non-orientable case. 

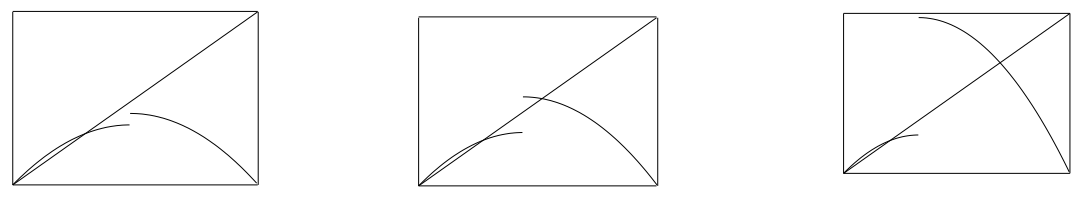

Figure 4 . Maps in the three easy cases of Lemma 4.

Lemma 5. (The non-orientable case - class $C$ ) Let $f: I \rightarrow I$ be in class $C$ and let $X_{2}$ denote the period two points of $f$ in with one point on each orbit in $x \leq c$ and the other in $x \geq c$. Let $X_{+}^{1,2}$ denote the fixed points and points on orbits of period two (possibly empty) in $x \geq c$ and $X_{-}^{1,2}$ denote the fixed points and points on orbits of period two (possibly empty) in $x \leq c$.

(i) If $f\left(c_{-}\right) \geq c$ and $f\left(c_{+}\right) \leq c$ then

(1) $\left(k_{+}, k_{-}\right)=\left((+1-1)^{\infty},(-1+1)^{\infty}\right)$;

(2) $I(x)=(-1+1)^{\infty}$ for all $x<c$ and $I(x)=(+1-1)^{\infty}$ for all $x>c$;

(3) $\Omega(f)=X_{2}$.

(ii) If $f\left(c_{-}\right) \leq c, f^{2}\left(c_{-}\right) \leq c, f\left(c_{+}\right)<c$ and $f^{2}\left(c_{+}\right)<c$ then

(1) $\left(k_{+}, k_{-}\right)=\left((+1)(-1)^{\infty},(-1)^{\infty}\right)$;

(2) $I(x)=(+1)(-1)^{\infty}$ for all $x>c$ and $I(x)=(-1)^{\infty}$ if $x<c$;

(3) $\Omega(f)=X_{-}^{1,2}$.

(iii) If $f\left(c_{-}\right) \leq c, f^{2}\left(c_{-}\right) \leq c, f\left(c_{+}\right)<c$ and $f^{2}\left(c_{+}\right)>c$ then

(1) $\left(k_{+}, k_{-}\right)=\left((+1-1)^{\infty},(-1)^{\infty}\right)$;

(2) $I(x)=(+1-1)^{\infty}$ for all $x>c$ and if $x<c$ then either $I(x)=(-1+1)^{\infty}$ or $I(x)=(-1)^{\infty}$ or $I\left(x_{-}\right)=(-1+1)^{\infty}$ and $I\left(x_{+}\right)=(-1)^{\infty}$;

(3) $\Omega(f)=X 2 \cup X_{-}^{1,2}$.

(iv) If $f\left(c_{-}\right) \leq c, f^{2}\left(c_{-}\right) \leq c, f\left(c_{+}\right) \geq c$ and $f^{2}\left(c_{+}\right) \geq c$ then

(1) $\left(k_{+}, k_{-}\right)=\left((+1)^{\infty},(-1)^{\infty}\right)$;

(2) $I(x)=(+1)^{\infty}$ or $I(x)=(+1)(-1)^{\infty}$ for all $x>c$ and if $x<c$ then either $I(x)=(-1)^{\infty}$ or $I(x)=(-1)(+1)^{\infty}$;

(3) $\Omega(f)=X_{-}^{1,2} \cup X_{+}^{1,2}$. 

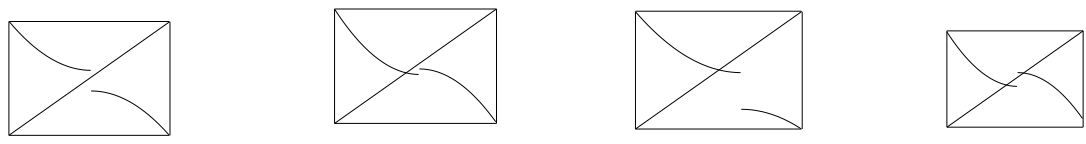

Figure 5. Maps in the first four easy cases of Lemma 5.

(v) If $f\left(c_{+}\right) \geq c f^{2}\left(c_{+}\right) \geq c, f\left(c_{-}\right)>c$ and $f^{2}\left(c_{-}\right)>c$ then the situation is as in case (ii) above, but with the roles of $x<c$ and $x>c$ reversed.

(vi) If $f\left(c_{+}\right) \geq c f^{2}\left(c_{+}\right) \geq c, f\left(c_{-}\right)>c$ and $f^{2}\left(c_{-}\right)<c$ then the situation is as in case (iii) above, but with the roles of $x<c$ and $x>c$ reversed.

Having got these simple cases out of the way we can consider the possibility of more complicated sequences.

\section{ITINERARIES AND KNEADING INVARIANTS: CASE A}

An admissible sequence for a map $f$ is a sequence which is the upper or lower itinerary of a point. In the cases not treated in the previous section, either the map is chaotic, or the first few iterates of $c$ define an invariant interval on this all the interesting dynamics occurs (which may be chaotic itself). Itineraries of points in this invariant interval are defined via the kneading invariants of the map. Different cases need to be considered separately, but all the proofs follow the usual arguments for unimodal maps as in Collet and Eckmann [10].

Lemma 6. (Class $A)$ Let $f: I \rightarrow I$ be an orientable msdc map with $f\left(c_{+}\right)>c$ and $f\left(c_{-}\right)<c$ and the interval $\left[f\left(c_{+}\right), f\left(c_{-}\right)\right]$is invariant. Let the kneading invariant of $f$ be $\left(k_{+}, k_{-}\right)$, then for all $x \in\left(f\left(c_{+}\right), f\left(c_{-}\right)\right)$and $r \geq 0$

$$
\sigma k_{+} \leq \sigma^{r} I\left(x_{ \pm}\right) \leq \sigma k_{-}
$$

Proof: Since $f^{r}\left(x_{ \pm}\right) \in\left[f\left(c_{+}\right), f\left(c_{-}\right)\right]$for all $r$ (where limits are taken through points which which are not preimages of $c$

$$
f\left(c_{+}\right) \leq f^{r}\left(x_{ \pm}\right) \leq f\left(c_{-}\right)
$$

and the result follows from Lemma 1.

The next step is to establish which pairs of sequences can be kneading sequences, and whether (25) is sufficient as well as necessary. 
Proposition 7. Let $k_{+}=(+1) \ldots$ and $k_{-}=(-1) \ldots$ be sequences which satisfy either

$$
\sigma k_{+} \leq \sigma^{r} k_{+} \leq \sigma k_{-} \text {and } \sigma k_{+}<\sigma^{r} k_{-} \leq \sigma k_{+}
$$

or

$$
\sigma k_{+}<\sigma^{r} k_{+} \leq \sigma k_{-} \text {and } \sigma k_{+} \leq \sigma^{r} k_{-}<\sigma k_{+}
$$

or

$$
k_{+}=\left(w_{+} w_{-}\right)^{\infty}, \quad k_{-}=\left(w_{-} w_{+}\right)^{\infty}
$$

where $w_{+}$and $w_{-}$are finite minimal words such that

$$
\sigma k_{+} \leq \sigma^{r} k_{ \pm} \leq \sigma k_{-}
$$

for all $r=0,1,2, \ldots$ then there exists a msdc map $f$ in class $A$ with $k(f)=\left(k_{+}, k_{-}\right)$.

Conversely, if $\left(k_{+}, k_{-}\right)$is the kneading invariant of a map $f$ then the pair will satisfy one of the three conditions above or it is one of the simple cases of Lemma 3.

Note: If the a msdc map has positive topological entropy then the standard techniques of [18] provides a semi-conjugacy of the map to a linear msdc map with slope equal to the logarithm of entropy. However, this linear map does not necessarily have the same kneading invariant as the original map (some intervals may be mapped to points under the semi-conjugacy). Hubbard and Sparrow [16] prove the existence of an expanding map for kneading invariants satisfying

$$
\sigma k_{+} \leq \sigma^{r} k_{+}<\sigma k_{-} \text {and } \sigma k_{+}<\sigma^{r} k_{-}<\sigma k_{-}
$$

by embedding points in the doubling map and then contracting intervals to points in an appropriate way. Unfortunately, this only works when left and right endpoints of the intervals to be contracted do not touch (as this leads to isolated points, and they require the set which is left to be homeomorphic to an interval). We use a modification to overcome this, and end up expanding intervals as in the construction of a Denjoy counterexample rather that contracting.

Proof: Define

$$
p(\mathbf{a})=\sum_{0}^{\infty} \frac{a_{i}}{2^{i+1}}
$$

and then

$$
P(\mathbf{a})= \begin{cases}p(\mathbf{a})-p\left(k_{+}\right) & \text {if } a_{1}=1 \\ p(\mathbf{a})-p\left(k_{-}\right) & \text {if } a_{1}=0\end{cases}
$$

so $P\left(k_{-}\right)=P\left(k_{+}\right)=0$ and $\mathbf{a} \prec_{o} \mathbf{b}$ implies $P(\mathbf{b})>P(\mathbf{a})$ unless $\mathbf{a}=A k_{-}$and $\mathbf{b}=A k_{+}$ in which case $P(\mathbf{b})=P(\mathbf{a})$.

If $\left(k_{+}, k_{-}\right)$satisfies $(27)$ then define $F$ on images of $x=0$ by

$$
F\left(P\left(\sigma^{n} k_{ \pm}\right)\right)=P\left(\sigma^{n+1} k_{ \pm}\right)
$$


Since the shift map is continuous at accumulation points of $\sigma^{k} k_{ \pm}, F$ can be extended to a map of the interval $\left[P\left(\sigma k_{+}\right), P\left(\sigma k_{-}\right)\right]$which is continuous and strictly increasing on the intervals $\left[P\left(\sigma k_{+}\right), 0\right)$ and $\left(0, P\left(\sigma k_{-}\right)\right]$, i.e. it is a map in class A. Moreover, $k(F)=\left(k_{+}, k_{-}\right)$follows easily.

Now suppose that $\sigma^{n} k_{+}=k_{-}$for some $n>0$, but $\sigma^{r} k_{-} \neq k_{+}$for all $r=0,1,2, \ldots$ i.e.

$$
\sigma k_{+} \leq \sigma^{r} k_{+} \leq \sigma k_{-} \text {and } \sigma k_{+} \leq \sigma^{r} k_{-}<\sigma k_{+}
$$

with equality in the second inequality of the first pair of inequalities for some $r$. Then $k_{+}=A k_{-}$, but the method used in the first part of this proof yields a Lorenz map with $k_{+}=A^{\infty}$. To get round this we need to create a homterval to the left of the discontinuity. Consider the interval $\left[p\left(\sigma k_{+}\right), p\left(\sigma k_{-}\right)\right]$, with $p$ as defined in (32) and note that

$$
p(\sigma \mathbf{a})= \begin{cases}2 p(\mathbf{a})-1 & \text { if } a_{0}=1 \\ 2 p(\mathbf{a})+1 & \text { if } a_{0}=-1\end{cases}
$$

Let $x_{m}=p\left(\sigma^{m} k_{-}\right), m=0,1,2, \ldots$, and for $m=-1,-2, \ldots$ let $X_{0}=\left\{x_{0}\right\}$ and let $X_{m}$ denote the set of points in $\left[p\left(\sigma k_{+}\right), p\left(\sigma k_{-}\right)\right]$which and map to $X_{m+1}$ under (36) and which are not in $X_{r}, r \geq m+1$, i.e. the preimages of $x_{0}$. Let $e_{m}=\varepsilon / 3^{|m|}$ for some small $\varepsilon>0$ and define a map $q$ from the images $x_{n}$ and preimages $y_{i, n} \in X_{n}$ of $x_{0}$ to a subset of the real line by

$$
\begin{aligned}
q\left(x_{m}\right) & =\left[p\left(x_{m}\right)+E\left(x_{m}\right)-e_{m}, p\left(x_{m}\right)+E\left(x_{m}\right)\right], \quad m=0,1,2, \ldots \\
q\left(y_{i, n}\right) & =\left[p\left(y_{i, m}\right)+E\left(y_{i, m}\right)-e_{m}, p\left(y_{i, m}\right)+E\left(y_{i, m}\right)\right], \quad m=-1,-2, \ldots \\
q\left(\sigma^{s} k_{+}\right) & \left.=p\left(\sigma^{s} k_{+}\right)+E_{(} \sigma^{s} k_{+}\right) \quad s=0,1, \ldots, n-1 \\
q\left(\sigma^{n} k_{+}\right) & =p\left(k_{-}\right)+E_{0}-\frac{1}{2} e_{0}
\end{aligned}
$$

where

$$
E(x)=\sum e_{k}
$$

and the sum is over all $k \geq 0$ such that $x_{k}<x$ and $(i, k)$ with $k<0$ such that $y_{i, k}<x$. Note that if $k_{-}$is periodic the sums need to be interpreted so that each point is only counted once (by setting an upper limit of the period $p$ minus one in the sum for $E$, and similarly limiting the range of $r$ and $s$ in (37)).

For each point $x$ for which $q$ is defined let

$$
Q(x)= \begin{cases}x-b_{0} & \text { if } x \leq b_{0} \\ x-q\left(k_{+}\right) & \text {if } x \geq q\left(k_{+}\right)\end{cases}
$$

and let $Q\left(q\left(\sigma^{r} k_{-}\right)\right)=I_{r}=\left[a_{r}, b_{r}\right]$ and $Q\left(y_{i, k}\right)=J_{i, k}$. Define $F$ on these points by

$$
F(x)= \begin{cases}Q\left(q\left(\sigma^{r+1} k_{+}\right)\right) & \text {if } r=0, \ldots, n-1 \\ f_{r}(x) & \text { if } x \in I_{r} \\ g_{i, k}(x) & \text { if } x \in J_{i, k}\end{cases}
$$


where $f_{r}: I_{r} \rightarrow I_{r+1}$ is a strictly increasing surjection with $f(x)>x$ on the interior of $I_{r}, f_{r}^{\prime}\left(a_{r}\right)>1$ and $f_{r}^{\prime}\left(b_{r}\right)<1$ so iterates of the map move towards the right hand end point of the interval $I_{r+1}$, and $g_{i, k}$ is similarly defined.

The function $F$ is now defined on a set of nonintersecting intervals and a finite set of points. Where it is defined, $x<y<0$ implies that $F(x)<F(y)$ and similarly for $x>0$. This can be extended to a msdc map in class A and $k(F)=\left(k_{+}, k_{-}\right)$.

In the final case, two finite sets of points need to be 'blown up' into intervals. The principle is precisely the same as the previous case and the details are omitted.

This exhausts all possibilities for class A maps, and the result is established with a minor amount of further thought.

\section{Itineraries AND KNEADing invariants: Classes B And C}

The methods for the other two cases are very similar to those of the previous section, complicated by parity arguments. We will not go through the full detailed proofs.

Lemma 8. (Class B) Let $f: I \rightarrow I$ be an semi-orientable msdc map in class $B$ (increasing in $x<c$ and decreasing in $x>c$ ) with $f\left(c_{-}\right)>c$.

(i) If $f\left(c_{-}\right) \geq f\left(c_{+}\right), f^{2}\left(c_{-}\right)<c$ and the interval $\left[f^{2}\left(c_{-}\right), f\left(c_{-}\right)\right]$is invariant then for all $x \in\left(f^{2}\left(c_{-}\right), f\left(c_{-}\right)\right)$and $r \geq 0$

$$
\begin{aligned}
\text { either } & \sigma^{2} k_{-} \preceq_{s o+} \sigma^{r} I\left(x_{ \pm}\right) \preceq_{s o+} k_{-} \\
\text {or } & k_{+} \preceq_{s o+} \sigma^{r} I\left(x_{ \pm}\right) \preceq_{s o+} \sigma k_{-}
\end{aligned}
$$

(ii) If $f\left(c_{-}\right)<f\left(c_{+}\right), f^{2}\left(c_{+}\right)<c$ and the interval $\left[f^{2}\left(c_{+}\right), f\left(c_{+}\right)\right]$is invariant then for all $x \in\left(f^{2}\left(c_{-}\right), f\left(c_{-}\right)\right)$and $r \geq 0$

$$
\begin{aligned}
\text { either } & \sigma^{2} k_{+} \preceq_{s o+} \sigma^{r} I\left(x_{ \pm}\right) \preceq_{s o+} k_{-} \\
\text {or } & k_{+} \preceq_{s o+} \sigma^{r} I\left(x_{ \pm}\right) \preceq_{s o+} \sigma k_{+}
\end{aligned}
$$

Lemma 9. (Class C) Let $f: I \rightarrow I$ be an non-orientable msdc map in class $C$ (decreasing in $x<c$ and $x>c$ ) with $f\left(c_{-}\right) \leq c$ and $, f^{2}\left(c_{-}\right) \leq c, f\left(c_{+}\right) \geq c$ and $f^{2}\left(c_{+}\right) \geq c$ then.

(i) If $f^{2}\left(c_{-}\right) \geq f\left(c_{+}\right)>c$ and the interval $\left[f\left(c_{-}\right), f^{2}\left(c_{-}\right)\right]$is invariant then for all $x \in\left(f\left(c_{-}\right), f^{2}\left(c_{-}\right)\right)$and $r \geq 0$

$$
\begin{aligned}
\text { either } & \sigma k_{-} \preceq_{n o} \sigma^{r} I\left(x_{ \pm}\right) \preceq_{n o} k_{-} \\
\text {or } & k_{+} \preceq_{n o} \sigma^{r} I\left(x_{ \pm}\right) \preceq_{n o} \sigma^{2} k_{-}
\end{aligned}
$$

(ii) If $f^{2}\left(c_{+}\right) \leq f\left(c_{-}\right)<c$ and the interval $\left[f^{2}\left(c_{+}\right), f\left(c_{+}\right)\right]$is invariant then for all $x \in\left(f^{2}\left(c_{+}\right), f\left(c_{+}\right)\right)$and $r \geq 0$

$$
\begin{aligned}
\text { either } & \sigma^{2} k_{+} \preceq_{n o} \sigma^{r} I\left(x_{ \pm}\right) \preceq_{n o} k_{-} \\
\text {or } & k_{+} \preceq_{n o} \sigma^{r} I\left(x_{ \pm}\right) \preceq_{n o} \sigma k_{+}
\end{aligned}
$$


In both cases the proofs follow from Lemma 1 in the standard way.

Now we come to the kneading invariants. Consider maps in class B with $f\left(c_{-}\right)>$ $f\left(c_{+}\right)$and an invariant interval $\left[f^{2}\left(c_{-}\right), f\left(c_{-}\right)\right], f^{2}\left(c_{-}\right)<c$. If for all $r>0$ for $k_{+}$and $r>2$ for $k_{-}$

$$
\text { either } \sigma^{2} k_{-} \prec_{s o+} \sigma^{r} k_{+} \prec_{s o+} k_{-} \text {or } k_{+} \preceq_{s o+} \sigma^{r} k_{+} \prec_{s o+} \sigma k_{-}
$$

and

$$
\text { either } \sigma^{2} k_{-} \preceq_{s o+} \sigma^{r} k_{-} \preceq_{s o+} k_{-} \text {or } k_{+} \prec_{s o+} \sigma^{r} k_{-} \preceq_{s o+} \sigma k_{-}
$$

then an argument similar to that for case A can be used to construct a msdc map $F$ in class B with $k(F)=\left(k_{+}, k_{-}\right)$. There are two slight differences: first we cannot use the addresses to construct the function $p$ as in (32), but need to transform the symbol sequences so that the lexicographical order replaces $\prec_{\text {sot }}$. This is easy (cf. [18]): simply replace $a_{k}$ by $b_{k}=\prod_{0}^{k} a_{k}$. The second thing to note is that if $\sigma^{p} k_{+}=k_{+}$ for some minimal $p>0$ then $k_{+}=w^{\infty}$ for a word $w$ of length $p$. If $w$ is even there is no complication, but if $w$ is odd then in constructing the map $F$ with $k(F)=\left(k_{+}, k_{-}\right)$ it is necessary to create a homterval to the right of $c$. A similar remark holds for $k_{-}$.

Suppose that $\sigma^{p} k_{+}=k_{-}$for some minimal $p>0$, so $k_{+}=w k_{-}$. Then provided

$$
\text { either } \sigma^{2} k_{-} \preceq_{s o+} \sigma^{r} k_{+} \preceq_{s o+} k_{-} \text {or } k_{+} \prec_{s o+} \sigma^{r} k_{+} \preceq_{s o+} \sigma k_{-}
$$

and

$$
\text { either } \sigma^{2} k_{-} \prec_{s o+} \sigma^{r} k_{-} \prec_{s o+} k_{-} \text {or } k_{+} \prec_{s o+} \sigma^{r} k_{-} \prec_{s o+} \sigma k_{-}
$$

there are again no problems except possibly the creation of homtervals if either $k_{-}=$ $w^{\infty}$ with $w$ odd and/or $k_{+}=w k_{-}$with $w$ even (of course, in the case of periodic sequences the homtervals are just basins of attraction of stable orbits). The case with $\sigma^{p} k_{-}=k_{+}$for some minimal $p>0$, with $\sigma^{r} k_{+} \neq k_{-}$a similar set of inequalities hold and the analysis is also similar.

This leaves the possibility that there exist (minimal) positive $p, q$ such that $\sigma^{p} k_{+}=$ $k_{-}$and $\sigma^{q} k_{-}=k_{+}$. This implies that $k_{+}=\left(w_{+} w_{-}\right)^{\infty}$ and $k_{-}=\left(w_{-} w_{+}\right)^{\infty}$. Again, there is no problem in constructing a map in class B with this kneading invariant if

$$
\text { either } \sigma^{2} k_{-} \prec_{s o+} \sigma^{r} k_{+} \prec_{s o+} k_{-} \text {or } k_{+} \prec_{s o+} \sigma^{r} k_{+} \prec_{s o+} \sigma k_{-}
$$

for $r=1, \ldots, p-1$ and

$$
\text { either } \sigma^{2} k_{-} \prec_{s o+} \sigma^{r} k_{-} \prec_{s o+} k_{-} \text {or } k_{+} \prec_{s o+} \sigma^{s} k_{-} \text {and } \sigma^{u} k_{-} \prec_{s o+} \sigma k_{-}
$$

for $r=3, \ldots, q-1, s=1, \ldots, q-1$ and $u=2, \ldots, q-1$, but if either $w_{-}$or $w_{+}$is odd a homterval needs to be created in the construction of the map $F$.

The limiting boundary case is $f\left(c_{-}\right)=f\left(c_{+}\right)$with $f\left(c_{ \pm}\right)<c$ and we assume that the interval $\left[f^{2}\left(c_{ \pm}\right), f\left(c_{ \pm}\right)\right]$is invariant. This is the case of the continuous unimodal map, and is very well studied. If $f^{n}\left(c_{ \pm}\right) \neq c, n \geq 1$ then the kneading sequences $k_{ \pm}$ differ only on their first symbol, and the sequence satisfies

$$
\text { either } \sigma^{2} k \prec_{s o+} \sigma^{r} k \prec_{s o+} k_{-} \text {or } k_{+} \prec_{s o+} \sigma^{r} k \prec_{s o+} \sigma k
$$


for $r=3,4, \ldots$ where $k$ can be either of $k_{ \pm}$. In this case our construction provides a map $F$ with $k(F)=\left(k_{+}, k_{-}\right)$. If $f^{p}\left(c_{ \pm}\right)=c$ for some minimal $p>1$ then define $w_{+}=+1 a$ and $w_{-}=-1 a$ then if $a$ is even, $k_{-}=(-1 a)^{\infty}$ and $k_{+}=1(a(-1))^{\infty}$ whilst if $a$ is odd then $k_{-}=-1(a 1)^{\infty}$ and $k_{-}=(1 a)^{\infty}$. Again, the method above yields a map $F$ with a homterval with $k(F)=\left(k_{+}, k_{-}\right)$.

Kneading invariants for maps in the second case of Lemma 8 can be treated similarly.

For maps in class $\mathrm{C}$ the way of coding orbits so that the lexicographical order is relevant is to use $b_{k}=(-1)^{k} a_{k}$ instead of the address, and this formulation is needed to construct the maps $F$ with appropriate kneading invariant, which, for the cases of Lemma 9, must satisfy

$$
\begin{aligned}
\text { either } & \sigma k_{-} \preceq_{n o} \sigma^{r} k_{ \pm} \preceq_{n o} k_{-} \\
\text {or } & k_{+} \preceq_{n o} \sigma^{r} k_{ \pm} \preceq_{n o} \sigma^{2} k_{-}
\end{aligned}
$$

or

$$
\begin{aligned}
\text { either } & \sigma^{2} k_{+} \preceq_{n o} \sigma^{r} k_{ \pm} \preceq_{n o} k_{-} \\
\text {or } & k_{+} \preceq_{n o} \sigma^{r} k_{ \pm} \preceq_{n o} \sigma k_{+}
\end{aligned}
$$

with the usual caveats when equalities hold.

\section{SUB-REGIONS AND RENORMALIZATION}

In this section we define sub-regions of the B-boxes and C-boxes in such a way that the boundary of chaos lies in these sub-regions and, more importantly, that the sub-regions map across each other in a well-defined way under the renormalization operations defined on each sub-region. This will enable us to define a subshift of finite type for the renormalization process in the next section. Although the various renormalizations have been defined elsewhere $[8,15]$, the choice of how the B-box and $\mathrm{C}$-boxes should be divided into sub-regions is not unique. The choices made below ensure that the way in which the regions maps across each other can be controlled.

Let $\left(f_{\mathbf{a}}\right)$ be a B-full family and without loss of generality set $c=0$. We define five sub-regions of the B-box together with four curves on the boundary of these regions and two points on the boundary of the B-box such that the boundary of chaos is in these regions. These definitions are given in terms of the end-points, $z_{0}$ and $z_{1}$, of the interval on which the map is defined and two points of period two: $y_{0}$ and $y_{1}$ with $y_{0}<0<y_{1}$ and $f\left(y_{i}\right)=y_{1-i}, i=0,1$. It is relatively straightforward to show that 
such points exist if $f_{\mathbf{a}}\left(0_{-}\right)>f_{\mathbf{a}}\left(0_{+}\right) \geq 0$ and $f_{\mathbf{a}}^{2}\left(0_{-}\right)<0$.

$$
\begin{aligned}
& B_{1}=\left\{\mathbf{a} \in \mathbb{R}^{2} \mid f_{\mathbf{a}}\left(0_{+}\right)<0\right\} \\
& L_{1}=\left\{\mathbf{a} \in \mathbb{R}^{2} \mid f_{\mathbf{a}}\left(0_{+}\right)=0\right\} \\
& B_{2}=\left\{\mathbf{a} \in \mathbb{R}^{2} \mid f_{\mathbf{a}}^{2}\left(0_{-}\right)<0,0<f_{\mathbf{a}}\left(0_{+}\right)<y_{1}\right\} \\
& L_{2}=\left\{\mathbf{a} \in \mathbb{R}^{2} \mid f_{\mathbf{a}}\left(0_{+}\right)=y_{1}\right\} \\
& B_{3}=\left\{\mathbf{a} \in \mathbb{R}^{2} \mid f_{\mathbf{a}}^{2}\left(0_{-}\right)<0, f_{\mathbf{a}}\left(0_{-}\right)>f_{\mathbf{a}}\left(0_{+}\right)>y_{1}\right\} \\
& L_{3}=\left\{\mathbf{a} \in \mathbb{R}^{2} \mid f_{\mathbf{a}}\left(0_{-}\right)=f_{\mathbf{a}}\left(0_{+}\right)\right\} \\
& B_{4}=\left\{\mathbf{a} \in \mathbb{R}^{2} \mid f_{\mathbf{a}}^{2}\left(0_{-}\right)<0, f_{\mathbf{a}}\left(0_{+}\right)>f_{\mathbf{a}}\left(0_{-}\right)>z_{1}\right\} \\
& L_{4}=\left\{\mathbf{a} \in \mathbb{R}^{2} \mid f_{\mathbf{a}}\left(0_{-}\right)=z_{1}\right\} \\
& B_{5}=\left\{\mathbf{a} \in \mathbb{R}^{2} \mid f_{\mathbf{a}}^{2}\left(0_{-}\right)<0, f_{\mathbf{a}}\left(0_{+}\right)>z_{1}>f_{\mathbf{a}}\left(0_{-}\right)\right\} \\
& S_{1}=\left\{\mathbf{a} \in \mathbb{R}^{2} \mid f_{\mathbf{a}}\left(0_{+}\right)=z_{0}, f_{\mathbf{a}}\left(0_{-}\right)=0\right\} \\
& S_{2}=\left\{\mathbf{a} \in \mathbb{R}^{2} \mid f_{\mathbf{a}}\left(0_{+}\right)>0, f_{\mathbf{a}}^{2}\left(0_{+}\right)=z_{0}, f_{\mathbf{a}}\left(0_{-}\right)=0\right\}
\end{aligned}
$$

From [15] it follows that the boundary of chaos is contained in these regions and stretches from $S_{1}$ to $S_{2}$; parameters not covered by these regions have trivial dynamics covered by Lemma 4 .

Similarly, if $\left(g_{\mathbf{b}}\right)$ is a full C-family with $c=0$ we can define seven sub-regions of the C-box and two special points, $T_{1}$ and $T_{2}$, such that the boundary of chaos in the C-box stretches from $T_{1}$ to $T_{2}$ and is contained entirely in the union of the regions. As in case $\mathrm{B}$, the definitions involve two points on the interval, $y_{0}<0<y_{1}$, with $g_{\mathbf{b}}\left(y_{i}\right)=y_{i}, i=0,1$. The fixed point $y_{0}$ exists if $g_{\mathbf{b}}\left(0_{-}\right)<0$ and $y_{1}$ exists if $g_{\mathbf{b}}\left(0_{+}\right)>0$.

$$
\begin{aligned}
& C_{1}=\left\{\mathbf{b} \in \mathbb{R}^{2} \mid g_{\mathbf{b}}\left(0_{+}\right)<z_{0}, g_{\mathbf{b}}\left(0_{-}\right)<0\right\} \\
& M_{1}=\left\{\mathbf{b} \in \mathbb{R}^{2} \mid g_{\mathbf{b}}\left(0_{+}\right)=z_{0}, g_{\mathbf{b}}\left(0_{-}\right)<0\right\} \\
& C_{2}=\left\{\mathbf{b} \in \mathbb{R}^{2} \mid z_{0}<g_{\mathbf{b}}\left(0_{+}\right)<0, g_{\mathbf{b}}\left(0_{-}\right)<0\right\} \\
& M_{2}=\left\{\mathbf{b} \in \mathbb{R}^{2} \mid g_{\mathbf{b}}\left(0_{+}\right)=0, g_{\mathbf{b}}\left(0_{-}\right)<0\right\} \\
& C_{3}=\left\{\mathbf{b} \in \mathbb{R}^{2} \mid g_{\mathbf{b}}\left(0_{+}\right)>0, g_{\mathbf{b}}\left(0_{-}\right)<0, g_{\mathbf{b}}^{2}\left(0_{-}\right)>y_{1}\right\} \\
& M_{3}=\left\{\mathbf{b} \in \mathbb{R}^{2} \mid g_{\mathbf{b}}\left(0_{+}\right)>0, g_{\mathbf{b}}\left(0_{-}\right)<0, g_{\mathbf{b}}^{2}\left(0_{-}\right)=y_{1}\right\} \\
& C_{4}=\left\{\mathbf{b} \in \mathbb{R}^{2} \mid g_{\mathbf{b}}\left(0_{+}\right)>0, g_{\mathbf{b}}^{2}\left(0_{+}\right)>y_{0}, g_{\mathbf{b}}\left(0_{-}\right)<0, g_{\mathbf{b}}^{2}\left(0_{-}\right)<y_{1}\right\} \\
& T_{1}=\left\{\mathbf{b} \in \mathbb{R}^{2} \mid g_{\mathbf{b}}\left(0_{-}\right)=g_{\mathbf{b}}\left(0_{+}\right)<0, g_{\mathbf{b}}\left(0_{-}\right)=g_{\mathbf{b}}^{3}\left(0_{-}\right)\right\} \\
& T_{2}=\left\{\mathbf{b} \in \mathbb{R}^{2} \mid g_{\mathbf{b}}\left(0_{-}\right)=g_{\mathbf{b}}\left(0_{+}\right)>0, g_{\mathbf{b}}\left(0_{+}\right)=g_{\mathbf{b}}^{3}\left(0_{+}\right)\right\}
\end{aligned}
$$

Three further regions, $C_{5}, C_{6}$ and $C_{7}$, and three curves, $M_{4}, M_{5}$ and $M_{6}$ are defined by symmetry $(x \rightarrow-x)$, where $C_{i}$ is the symmetric image of $C_{8-i}, i=5,6,7$, and $M_{j}$ is the symmetric image of $M_{7-j}, j=4,5,6$.

Similar regions, lines and points are defined for maps in class D, corresponding to the sets defined for class B after the transformation $x \rightarrow-x$. We shall not list these regions, curves and points here, they will be denoted by the symbols $D_{k}, k=1, \ldots, 5$, $N_{r}, r=1, \ldots, 4$, and $R_{s}, s=1,2$, respectively in the obvious way. 

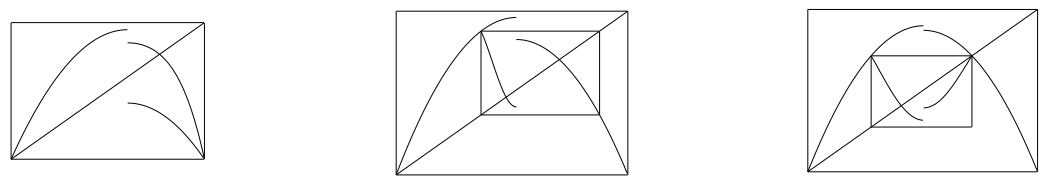

Figure 6 . Renormalized maps for $B_{1}, B_{2}$ and $B_{3}$.

The next step is to define induced maps for maps in each region, determine the range of behaviour that these induced maps can exhibit and associate to each renormalization operation a pair of functions which define the iterates of the induced map in terms of the iterates of the original map and the periodic orbits which are left behind by the renormalization.

Suppose that $\mathbf{a} \in B_{1}$, then $f_{\mathbf{a}}\left(\left(0, z_{1}\right]\right) \subseteq\left[z_{0}, 0\right)$ and so $f_{\mathbf{a}}^{2}$ restricted to $\left(0, z_{1}\right]$ is monotonic and decreasing (as $f_{\mathbf{a}}$ is decreasing in $x>0$ and increasing in $x<0$, so the the induced map $R_{B_{1}} f_{\mathbf{a}}: I \rightarrow I$ defined by

$$
R_{B_{1}} f_{\mathbf{a}}(x)= \begin{cases}f_{\mathbf{a}}(x) & \text { if } x<0 \\ f_{\mathbf{a}}^{2}(x) & \text { if } x>0\end{cases}
$$

is a map in class B. Moreover, $f_{\mathbf{a}}^{2}\left(0_{+}\right) \leq f_{\mathbf{a}}\left(0_{-}\right)$, with equality if $f_{\mathbf{a}}\left(0_{+}\right)=0$, and in the limit of $f_{\mathbf{a}}\left(0_{+}\right)=z_{0}$ (i.e. $\left.z_{1} \rightarrow 0\right), f_{\mathbf{a}}^{2}\left(0_{+}\right)=z_{0}$. Hence $R_{B_{1}} f_{\mathbf{a}}$ is in either $B_{1}, B_{2}$, $B_{3}, L_{1}$ or $L_{2}$ (see Figure 7 ). Furthermore, maps on the boundary of chaos in $B_{1}$ are mapped by $R_{B_{1}}$ across the boundary of chaos in $B_{1} \cup L_{1} \cup B_{2} \cup L_{2} \cup B_{3}$. In general, if the map $f_{\mathbf{a}}$ is itself an induced map defined by the $n_{1}^{\text {th }}$ iterate of a map in $x>0$ and the $n_{2}^{\text {th }}$ iterate of a map in $x<0$, then the induced map $R_{B_{1}} f_{\mathbf{a}}$ has iterates $\beta_{1}\left(n_{1}, n_{2}\right)$ where $\beta_{1}: \mathbb{N}^{2} \rightarrow \mathbb{N}^{2}$ is defined by

$$
\beta_{1}\left(n_{1}, n_{2}\right)=\left(n_{1}+n_{2}, n_{2}\right)
$$

Since the induced map is defined on the whole interval for which $f_{\mathbf{a}}$ is defined we do not lose any periodic orbits so we do not define any operator to give the period of periodic orbits left behind in this case. The important information is thus that the boundary of chaos in $B_{1}$ is mapped under renormalization to the boundary of chaos in $B_{1} \cup L_{1} \cup B_{2} \cup L_{2} \cup B_{3}$ and the value of $\beta_{1}$, which gives the iterates of the induced map. 
Now consider $\mathbf{a} \in B_{2}$. By the remarks already made (above the definitions of the regions $\left.B_{i}\right)$ there is an orbit of period two $\left\{y_{0}, y_{1}\right\}$ with $y_{0}<0<y_{1}$. Define the map $R_{B_{2}} f_{\mathbf{a}}:\left[y_{0}, y_{1}\right] \rightarrow I$ by

$$
R_{B_{2}} f_{\mathbf{a}}(x)= \begin{cases}f_{\mathbf{a}}^{3}(x) & \text { if } x<0 \\ f_{\mathbf{a}}(x) & \text { if } x>0\end{cases}
$$

(see Figure 7 ). It is fairly easy to see that this maps $\left[y_{0}, y_{1}\right]$ into itself provided $f_{\mathbf{a}}^{3}\left(0_{-}\right) \geq y_{0}$ and that if $f_{\mathbf{a}}^{3}\left(0_{-}\right)<y_{0}$ then the map has positive topological entropy. Moreover, $R_{B_{2}} f_{\mathbf{a}}$ is in class C. Varying $\mathbf{a}$ in $B_{2}$ it should be clear that we can obtain any map on the boundary of chaos in $\mathrm{C}$ with $g_{\mathbf{b}}\left(0_{+}\right)>0$. Hence the boundary of chaos in $B_{2}$ is mapped under renormalization to the part of the boundary of chaos in class $\mathrm{C}$ contained in $C_{3} \cup M_{3} \cup C_{4} \cup M_{4} \cup C_{5} \cup M_{5} \cup C_{6} \cup M_{6} \cup C_{7}$.

The effect of this renormalization on iterates of a general induced map is given by

$$
\beta_{2}\left(n_{1}, n_{2}\right)=\left(n_{1}, n_{1}+2 n_{2}\right)
$$

The only recurrent dynamics which is not contained in the union of forward iterates of $\left(y_{0}, y_{1}\right)$ consists of fixed points $\left(z_{0}\right)$ which we knew about already (from the definition of maps in class B) and so the new information is that there is an orbit of period two. More generally, an orbit of period $n_{1}+n_{2}$ is 'left behind' by this renormalization step. Hence we define $p_{B_{2}}: \mathbb{N}^{2} \rightarrow \mathbb{N}$ by

$$
p_{B_{2}}\left(n_{1}, n_{2}\right)=n_{1}+n_{2}
$$

Rather than work painfully through the remaining cases, the idea should be clear enough by now and the results are described in Table 1 (the renormalization in region $B_{3}$ is sketched in Figure 7 ). Note that the only subtle aspect of this paper is the second column of this table. The sub-regions of the boxes must be defined in such a way that the boundary of chaos in one region is mapped onto the boundary of chaos in a union of other sub-regions with no remainder. As mentioned earlier this is not a unique representation, but captures the dynamics with a minimum of fuss.

\section{A subshift of Finite type Under Renormalization}

The first two columns of Table 1 define a subshift of finite type for the renormalization of these single discontinuity maps: given (almost) any sequence of symbols allowed by these columns there is a map on the boundary of chaos which can be renormalized with this sequence of operations. Maps which renormalize to class A may be further renormalizable, but since these transitions are well understood $[11,19]$ we will ignore them here. Now, for maps which do not fall into class A under renormalization there are two possibilities: either they can be renormalized a finite number of times and then fall on the special points $S_{k}, T_{k}$ or $R_{k}(k=1,2)$, defined in the previous section, or they can be renormalized an infinite number of times without falling on these points. For maps on the boundary of chaos in the former case there is a finite 
TABLE 1. Effect of renormalization in the different regions described in the text. The image is the set of regions mapped onto by renormalization, the renormalization itself gives the induced map as iterates on the right and left respectively as a function of the original right and left iterates, and the period indicates the period of periodic orbits 'left behind' by renormalization.

\section{Region Image $\quad$ Renormlization $\quad$ Period}

\begin{tabular}{|c|c|c|c|}
\hline$B_{1}$ & $B_{1} \cup L_{1} \cup B_{2} \cup L_{2} \cup B_{3}$ & $\beta_{1}\left(n_{1}, n_{2}\right)=\left(n_{1}+n_{2}, n_{2}\right)$ & $\emptyset$ \\
\hline$L_{1}$ & $M_{2}$ & $\lambda_{1}\left(n_{1}, n_{2}\right)=\left(n_{1}, n_{1}+2 n_{2}\right)$ & $n_{1}+n_{2}$ \\
\hline$B_{2}$ & $\begin{array}{c}C_{3} \cup C_{4} \cup C_{5} \cup C_{6} \cup C_{7} \\
\cup M_{3} \cup M_{4} \cup M_{5} \cup M_{6}\end{array}$ & $\beta_{2}\left(n_{1}, n_{2}\right)=\left(n_{1}, n_{1}+2 n_{2}\right)$ & $n_{1}+n_{2}$ \\
\hline$L_{2}$ & $N_{4}$ & $\lambda_{2}\left(n_{1}, n_{2}\right)=\left(2 n_{1}, n_{1}+n_{2}\right)$ & $n_{1}$ \\
\hline$B_{3}$ & $D_{4}$ & $\beta_{3}\left(n_{1}, n_{2}\right)=\left(2 n_{1}, n_{1}+n_{2}\right)$ & $n_{1}$ \\
\hline$L_{3}$ & $N_{3}$ & $\lambda_{3}\left(n_{1}, n_{2}\right)=\left(2 n_{1}, n_{1}+n_{2}\right)$ & $n_{1}$ \\
\hline$B_{4}$ & $D_{1} \cup N_{1} \cup D_{2} \cup N_{3} \cup D_{3}$ & $\beta_{4}\left(n_{1}, n_{2}\right)=\left(2 n_{1}, n_{1}+n_{2}\right)$ & $n_{1}$ \\
\hline$L_{4}$ & $R_{3}$ & $\lambda_{4}\left(n_{1}, n_{2}\right)=\left(2 n_{1}, n_{1}+n_{2}\right)$ & $n_{1}$ \\
\hline$B_{5}$ & $A$ & $\beta_{5}\left(n_{1}, n_{2}\right)=\left(2 n_{1}, n_{2}\right)$ & $n_{1}$ \\
\hline$C_{1}$ & $A$ & $\gamma_{1}\left(n_{1}, n_{2}\right)=\left(n_{1}+n_{2}, 2 n_{2}\right)$ & $n_{2}$ \\
\hline$M_{1}$ & $S_{1}$ & $\mu_{1}\left(n_{1}, n_{2}\right)=\left(n_{1}, 2 n_{2}\right)$ & $n_{2}$ \\
\hline$C_{2}$ & $B_{1}$ & $\gamma_{1}\left(n_{1}, n_{2}\right)=\left(n_{1}, 2 n_{2}\right)$ & $n_{2}$ \\
\hline$M_{2}$ & $L_{1}$ & $\mu_{2}\left(n_{1}, n_{2}\right)=\left(n_{1}, 2 n_{2}\right)$ & $n_{2}$ \\
\hline$C_{3}$ & $B_{2} \cup L_{2} \cup B_{3} \cup L_{3} \cup B_{4}$ & $\gamma_{3}\left(n_{1}, n_{2}\right)=\left(n_{1}, 2 n_{2}\right)$ & $n_{2}$ \\
\hline$M_{3}$ & $S_{2}$ & $\mu_{3}\left(n_{1}, n_{2}\right)=\left(n_{1}, 2 n_{2}\right)$ & $n_{2}$ \\
\hline$C_{4}$ & $A$ & $\gamma_{4}\left(n_{1}, n_{2}\right)=\left(2 n_{1}, 2 n_{2}\right)$ & $\left\{n_{1}, n_{2}\right\}$ \\
\hline$M_{4}$ & $R_{2}$ & $\mu_{4}\left(n_{1}, n_{2}\right)=\left(2 n_{1}, n_{2}\right)$ & $n_{1}$ \\
\hline$C_{5}$ & $D_{2} \cup N_{2} \cup D_{3} \cup N_{3} \cup D_{4}$ & $\gamma_{4}\left(n_{1}, n_{2}\right)=\left(2 n_{1}, n_{2}\right)$ & $n_{1}$ \\
\hline$M_{5}$ & $N_{1}$ & $\mu_{5}\left(n_{1}, n_{2}\right)=\left(2 n_{1}, n_{2}\right)$ & $n_{1}$ \\
\hline$C_{6}$ & $D_{1}$ & $\gamma_{6}\left(n_{1}, n_{2}\right)=\left(2 n_{1}, n_{2}\right)$ & $n_{1}$ \\
\hline$M_{6}$ & $R_{1}$ & $\mu_{6}\left(n_{1}, n_{2}\right)=\left(2 n_{1}, n_{2}\right)$ & $n_{1}$ \\
\hline$C_{7}$ & $A$ & $\gamma_{7}\left(n_{1}, n_{2}\right)=\left(2 n_{1}, n_{1}+n_{2}\right)$ & $n_{1}$ \\
\hline$D_{1}$ & $D_{1} \cup N_{1} \cup D_{2} \cup N_{2} \cup D_{3}$ & $\delta_{1}\left(n_{1}, n_{2}\right)=\left(n_{1}, n_{1}+n_{2}\right)$ & $\emptyset$ \\
\hline$N_{1}$ & $M_{5}$ & $\nu_{1}\left(n_{1}, n_{2}\right)=\left(2 n_{1}+n_{2}, n_{2}\right)$ & $n_{1}+n_{2}$ \\
\hline$D_{2}$ & $\begin{array}{l}C_{1} \cup C_{2} \cup C_{3} \cup C_{4} \cup C_{5} \\
\cup M_{1} \cup M_{2} \cup M_{3} \cup M_{4}\end{array}$ & $d e_{2}\left(n_{1}, n_{2}\right)=\left(2 n_{1}+n_{2}, n_{2}\right)$ & $n_{1}+n_{2}$ \\
\hline$N_{2}$ & $L_{3}$ & $\nu_{2}\left(n_{1}, n_{2}\right)=\left(n_{1}+n_{2}, 2 n_{2}\right)$ & $n_{2}$ \\
\hline$D_{3}$ & $B_{4}$ & $\delta_{3}\left(n_{1}, n_{2}\right)=\left(n_{1}+n_{2}, 2 n_{2}\right)$ & $n_{2}$ \\
\hline$N_{3}$ & $L_{3}$ & $\nu_{3}\left(n_{1}, n_{2}\right)=\left(n_{1}+n_{2}, 2 n_{2}\right)$ & $n_{2}$ \\
\hline$D_{4}$ & $B_{1} \cup L_{1} \cup B_{2} \cup L_{3} \cup B_{3}$ & $\delta_{4}\left(n_{1}, n_{2}\right)=\left(n_{1}+n_{2}, 2 n_{2}\right)$ & $n_{2}$ \\
\hline$N_{4}$ & $S_{1}$ & $\nu_{4}\left(n_{1}, n_{2}\right)=\left(n_{1}+n_{2}, 2 n_{2}\right)$ & $n_{2}$ \\
\hline$D_{5}$ & $A$ & $\delta_{5}\left(n_{1}, n_{2}\right)=\left(n_{1}, 2 n_{2}\right)$ & $n_{2}$ \\
\hline
\end{tabular}


set of periods, and the transition from zero entropy to positive entropy involves a discontinuous jump in the number of periods present in the maps. In the latter case the maps can be renormalized an infinite number of times, leaving behind an infinite set of periodic orbits. It is this case that we pursue further here. Thus, in what follows, we ignore the boundary elements $L_{i}, M_{k}$ and $N_{i}$, and concentrate on maps which are infinitely renormalizable and which never renormalize to these boundaries. These ignored cases are easy to reintroduce into the analysis using Table 1.

Since class $\mathrm{A}$ is also to be ignored, we can ignore $B_{5}, C_{1}, C_{4}, C_{7}$ and $D_{5}$ on the grounds that any map which falls into one of these classes under renormalization then renormalizes to class A. The graph associated with the remaining regions is shown in Figure 8: the arrows indicating the allowed paths. Note that the diagram is symmetric under the transformation.

$$
\left(B_{i}, C_{2}, C_{3}, C_{5}, C_{6}, D_{i}\right) \rightarrow\left(D_{i}, C_{6}, C_{5}, C_{3}, C_{2}, B_{i}\right)
$$

Once again, this simply reflects the relationships between the maps under the transformation $x \rightarrow-x$.

We now have to be a little careful: since the renormalization map is discontinuous, there may be the (inevitable) ambiguity about whether a sequence allowed by the subshift actually corresponds to a map inside the region, or whether it reflects the behaviour on the boundary of the region. The two fixed points of this subgraph $B_{1}^{\infty}$ and $D_{1}^{\infty}$, are a case in point, since they represent the points $R_{1}$ and $S_{1}$ respectively. Indeed any sequence allowed by this diagram which ends with an infinite sequence of $B_{1} \mathrm{~s}$ or $D_{1} \mathrm{~s}$ corresponds to the case mentioned above for which there is a finite set of periods for maps on the boundary of chaos. There are two pairs of periodic orbits of period two allowed by the subgraph of Figure 8: $\left(D_{3} B_{4}\right)^{\infty}$ and its symmetric image $\left(B_{3} D_{4}\right)^{\infty}$ correspond to period-doubling. In the context of the discontinuous maps defined here this sequence of bifurcations is unstable to perturbations of the maps although in the context of continuous maps of the intervals (for class $\mathrm{B}$ or $\mathrm{D}$ ) or symmetric maps in class $\mathrm{C}$ this route is stable to perturbations. Thus we identify these sequences with the boundary orbit $\left(L_{3} N_{3}\right)^{\infty}$. Looking at the remainder of possible boundary behaviour we see that any other sequence (i.e. one which does not contain the four infinite sequences described above) does indeed correspond to an orbit of the subshift which is not on the boundary at any stage of renormalization.

Thus sequences which contain the subsequences $B_{1}^{\infty}, D_{1}^{\infty},\left(D_{3} B_{4}\right)^{\infty}$ and $\left(B_{3} D_{4}\right)^{\infty}$ are not allowed. (Alternatively we could have worked with closed regions, in which case a subshift is obtained for which all sequences are allowed but some must be identified.)

The second pair of periodic cycles of period two is $\left(C_{3} B_{2}\right)^{\infty}$ and $\left(C_{5} D_{2}\right)^{\infty}$. These correspond to maps which undergo the anharmonic route to chaos described in $[13,14]$ and is robust to small perturbations of the defining families. So any sequence allowed by Figure 8 which ends with either of these sequences defines a line segment in 


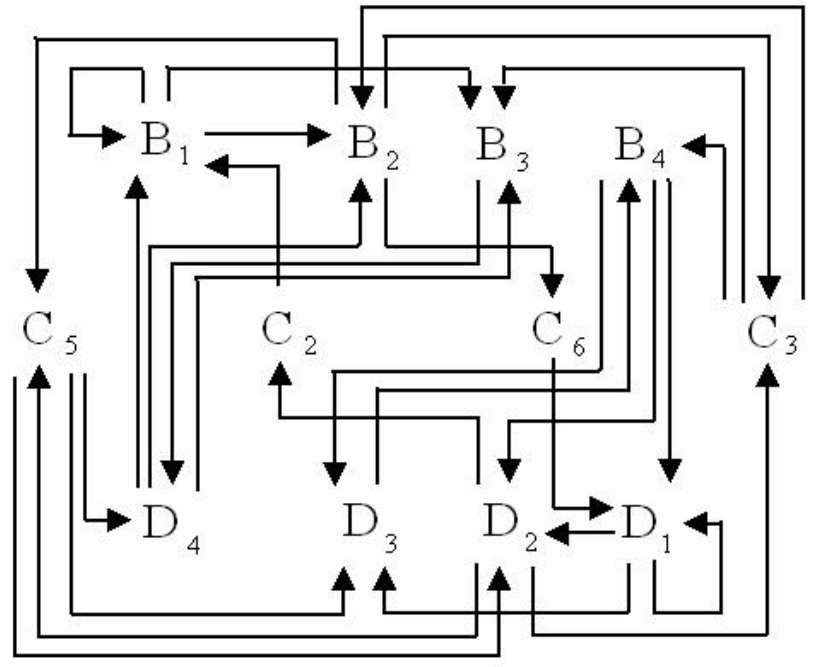

FiguRE 7. Directed graph for the subshift defined by renormalization of monotonic maps with a single discontinuity. All infinite paths allowed by the graph except those containing the sequences $D_{1}^{\infty}, B_{1}^{\infty}$, $\left(B_{3} D_{4}\right)^{\infty}$ and $\left(D_{3} B_{4}\right)^{\infty}$ correspond to maps on the boundary of chaos.

parameter space on which maps undergo the anharmonic route to chaos after some initial 'out of sequence' set of bifurcations. We believe that these are the only line segments on the boundary of chaos which do not lie in A-boxes.

Figure 8 together with Table 1 gives a complete catalogue of the periods and renormalizations of maps on the boundary of chaos for the families of maps we are considering with one exception (described in section six). In the remainder of this paper we consider two sets of possibilities in more detail: the low-period cycles of Figure 8 and the anomalous anharmonic routes to chaos. 


\section{LOW-PERIOD ORBITS OF THE SUBSHIFT}

When describing low order periodic orbits of the subshift we shall adopt the convention that sequences are read from left to right (as though they were maps acting on the left), so $\left(B_{3} D_{4} B_{1}\right)^{\infty}$ is an allowed sequence whilst $\left(B_{1} D_{4} B_{3}\right)^{\infty}$ is not. Since the subshift is symmetric, periodic sequences either come in pairs or they are themselves symmetric. We shall consider the periodic sequences of length two and three together with the symmetric cycle of length four and six.

\section{Cycles of period two and symmetric cycles of period four}

There are two independent cycles of period two allowed by the graph in Figure 8: the cycles $\left(C_{3} B_{2}\right)^{\infty}$ and $\left(C_{5} D_{2}\right)^{\infty}$. Maps on the boundary of chaos in these cases have been described elsewhere $[13,14]$ and will be considered in the next section. There is one symmetric cycle of period four: $\left(C_{3} B_{2} C_{5} D_{2}\right)^{\infty}$, and it is this case that we concentrate on here.

Suppose that some map $g \in C_{3}$ is infinitely renormalizable with a sequence of renormalizations corresponding to this renormalization cycle. Then there is a natural period in the renormalization process: after four renormalizations we come back and repeat the same renormalizations. So, suppose that after $4 \mathrm{k}$ renormalization steps we have a renormalized map in $C_{3}$ involving the iterates $\left(n_{1}, n_{2}\right)$. Applying $\gamma_{3}$ we obtain a new map in $B_{2}$ with iterates $\left(n_{1}, 2 n_{2}\right)$ and leave behind an orbit of period $n_{2}$. Now applying $\beta_{2}$ we get a new map in $C_{5}$ with iterates $\left(n_{1}, n_{1}+4 n_{2}\right)$ and an orbit of period $n_{1}+2 n_{2}$. Applying $\gamma_{5}$ gives a map in $D_{2}$ with iterates $\left(2 n_{1}, n_{1}+4 n_{2}\right)$ and an orbit of period $n_{1}$. Finally, applying $\delta_{2}$ we get a map with iterates $\left(5 n_{1}+4 n_{2}, n_{1}+4 n_{2}\right)$, an orbit of period $3 n_{1}+4 n_{2}$ and we are back where we started. Hence, through one renormalization cycle the iterates of the induced map changes from $\left(n_{1}, 2 n_{2}\right)$ to $\left(5 n_{1}+4 n_{2}, n_{1}+4 n_{2}\right)$ and periodic orbits of period $n_{2}, n_{1}, n_{1}+2 n_{2}$ and $3 n_{1}+4 n_{2}$ have been left behind. This can be represented conveniently in the following way: after $4 k$ renormalization steps the iterates of the induced map are $\left(n_{1}^{k}, n_{2}^{k}\right)$ where

$$
\left(\begin{array}{l}
n_{1}^{k+1} \\
n_{2}^{k+1}
\end{array}\right)=A\left(\begin{array}{l}
n_{1}^{k} \\
n_{2}^{k}
\end{array}\right)
$$

with

$$
A=\left(\begin{array}{ll}
5 & 4 \\
1 & 4
\end{array}\right)
$$

and the periodic orbits created in the four renormalizations between the $4 k^{\text {th }}$ and $4(k+1)^{t h}$ are $p_{4 k+1}, \ldots, p_{4 k+4}$ where

$$
\left(\begin{array}{l}
p_{4 k+1} \\
p_{4 k+2} \\
p_{4 k+3} \\
p_{4 k+4}
\end{array}\right)=B\left(\begin{array}{l}
n_{1}^{k} \\
n_{2}^{k}
\end{array}\right), \quad B=\left(\begin{array}{ll}
0 & 1 \\
1 & 0 \\
1 & 2 \\
3 & 4
\end{array}\right)
$$


and $n_{1}^{0}=n_{2}^{0}=1$. Applying these operations we find that this gives a map on the boundary of chaos with periodic orbits with periods

$$
1,1,3,7,5,9,19,47,29,65,123,311, \ldots
$$

Another route to chaos associated with this renormalization cycle can be found in class $B_{2}$, infinitely renormalizable with $\left(B_{2} C_{5} D_{2} C_{3}\right)^{\infty}$. In this case

$$
A=\left(\begin{array}{ll}
5 & 2 \\
2 & 4
\end{array}\right), \quad B=\left(\begin{array}{ll}
1 & 0 \\
1 & 1 \\
1 & 2 \\
3 & 2
\end{array}\right)
$$

giving the sequence of periods

$$
1,2,3,5,7,13,19,33,47,85,123,217, \ldots
$$

The remaining two permutations of the renormalization cycle give the symmetric images of these two routes and hence the same sequence of periods.

\section{Cycles of period three and symmetric cycles of period six}

There are two independent cycles of period three, $\left(B_{1} B_{3} D_{4}\right)^{\infty}$ and $\left(B_{4} D_{2} C_{3}\right)^{\infty}$ together with their symmetric images, which we shall ignore. There are also two symmetric cycles of period six, $\left(C_{5} D_{4} B_{2} C_{3} B_{4} D_{2}\right)^{\infty}$ and $\left(B_{2} C_{6} D_{1} D_{2} C_{2} B_{1}\right)^{\infty}$. The three routes to chaos associated with $\left(B_{1} B_{3} D_{4}\right)^{\infty}$ will be described in the same way as we approached the previous example. Since no periodic orbit is associated with a renormalization in $B_{1}$ each run through the cycle will produce two periodic orbits. After each run through the cycle the iterate of the induced map will be changed, so the matrix $A$ is defined as before (except it describes the change after three rather than four renormalization steps) whilst the matrix $B$ in now $2 \times 2$ and

$$
\left(\begin{array}{l}
p_{2 k+1} \\
p_{2 k+2}
\end{array}\right)=B A^{k}\left(\begin{array}{l}
1 \\
1
\end{array}\right)
$$

For $\left(B_{1} B_{3} D_{4}\right)^{\infty}$ (and hence maps in $\left.B_{1}\right)$ we find

$$
A=\left(\begin{array}{ll}
3 & 4 \\
2 & 4
\end{array}\right), \quad B=\left(\begin{array}{ll}
1 & 1 \\
1 & 2
\end{array}\right)
$$

giving the sequence

$$
2,3,13,19,83,121,529,771, \ldots
$$

For maps in $B_{3}$ which are infinitely renormalizable with $\left(B_{3} D_{4} B_{1}\right)^{\infty}$ we find

$$
A=\left(\begin{array}{ll}
5 & 3 \\
2 & 2
\end{array}\right), \quad B=\left(\begin{array}{ll}
1 & 0 \\
1 & 1
\end{array}\right)
$$

giving the sequence

$$
1,2,8,12,52,76,332,484, \ldots
$$


and for maps in $D_{4}$ which are infinitely renormalizable with $\left(D_{4} B_{1} B_{3}\right)^{\infty}$

$$
A=\left(\begin{array}{ll}
2 & 6 \\
1 & 5
\end{array}\right), \quad B=\left(\begin{array}{ll}
0 & 1 \\
1 & 3
\end{array}\right)
$$

giving the sequence

$$
1,4,6,24,38,166,242,1068, \ldots
$$

Maps which are infinitely renormalizable with $\left(B_{4} D_{2} C_{3}\right)^{\infty}$ and its permutations are described in precisely the same way, but now $B$ is a $2 \times 3$ matrix and the periodic orbits are in the sequence

$$
\left(\begin{array}{l}
p_{3 k+1} \\
p_{3 k+2} \\
p_{3 k+3}
\end{array}\right)=B A^{k}\left(\begin{array}{l}
1 \\
1
\end{array}\right)
$$

For maps in $B_{4}$ which are infinitely renormalizable with $\left(B_{4} D_{2} C_{3}\right)^{\infty}$

$$
A=\left(\begin{array}{ll}
5 & 1 \\
2 & 2
\end{array}\right), \quad B=\left(\begin{array}{ll}
1 & 0 \\
1 & 1 \\
3 & 1
\end{array}\right)
$$

giving periods

$$
1,2,4,5,10,22,34,54,122,190,298,678, \ldots
$$

For the corresponding maps in $D_{2}$

$$
A=\left(\begin{array}{ll}
4 & 2 \\
2 & 3
\end{array}\right), \quad B=\left(\begin{array}{ll}
0 & 1 \\
1 & 1 \\
2 & 1
\end{array}\right)
$$

giving the sequence of periods

$$
1,2,3,5,11,17,27,61,95,149,339,529, \ldots
$$

And for maps in $C_{2}$ we find

$$
A=\left(\begin{array}{ll}
5 & 2 \\
1 & 2
\end{array}\right), \quad B=\left(\begin{array}{ll}
0 & 1 \\
1 & 0 \\
3 & 2
\end{array}\right)
$$

giving

$$
1,1,5,3,7,27,13,41,149,67,231,827, \ldots
$$

The way to relate the subshift to the periodic orbits on the boundary of chaos should now be clear and we will not go through the full calculation for the two remaining cases, $\left(C_{5} D_{4} B_{2} C_{3} B_{4} D_{2}\right)^{\infty}$ and $\left(B_{2} C_{6} D_{1} D_{2} C_{2} B_{1}\right)^{\infty}$. However, just to emphasize the point that the sequences of periods are sometimes quite curious we end this section with the first few terms from the latter case:

$$
1,3,5,21,13,51,77,357,205,819,1229,5733, \ldots
$$




\section{The AnHARMonic Route to CHAOs: GENERALISATIONS}

The anharmonic route to chaos, described in $[13,14]$, is stable to perturbations and so we expect any sequence allowed by the subshift which ends $\left(B_{2} C_{3}\right)^{\infty}$ or $\left(D_{2} C_{5}\right)^{\infty}$ to correspond to segments of curves on the boundary of chaos in parameter space. Consider the case of $\left(B_{2} C_{3}\right)^{\infty}$, for example. In the notation of the previous section we find that

$$
A=\left(\begin{array}{ll}
1 & 0 \\
2 & 4
\end{array}\right), \quad B=\left(\begin{array}{ll}
1 & 1 \\
1 & 2
\end{array}\right)
$$

and so we obtain the sequence of periods

$$
2,3,7,13,27,53, \ldots
$$

or

$$
p_{n+1}=2 p_{n}+(-1)^{n}, \quad p_{1}=2
$$

Note the $n_{1}$ is not charged by this renormalization process, but that $f\left(0_{+}\right)>0$ since $f \in B_{2}$. Hence there is a fixed point (and possibly a point of period two) of $f$ in $x>0$ which is not picked out by the renormalization scheme. This is the one exception to the set of periods obtained from Figure 8 and Table 1 which must be introduced by hand. More generally, if there is some finite number of renormalization steps followed by $\left(B_{2} C_{3}\right)^{\infty}$ an orbit of period $n_{1}$ and, possibly, an orbit of period $2 n_{1}$, exist for the corresponding map on the boundary of chaos which will not appear in the list generated by the algorithm of section four.

As an example consider the allowed sequence $C_{2} B_{1}\left(B_{2} C_{3}\right)^{\infty}$. Applying the renormalization rules we find that there are periodic orbits of period one and $\left(p_{n}\right)$, with

$$
p_{n+1}=2 p_{n}+3(-1)^{n}, \quad p_{1}=5
$$

and that $n_{1}=3$ during the cycle $\left(B_{2} C_{3}\right)^{\infty}$. Hence the maps corresponding to this sequence also have an orbit of period three and, possibly, an orbit of period six. All preimages of $\left(B_{2} C_{3}\right)^{\infty}$ allowed by the subshift can be treated similarly.

\section{Rigorous statements}

The analysis of the past few sections is based on the intuitive description of renormalization given in section 7 . These can be made rigorous by looking at the possible renormalizations of kneading invariants on the symbolic level. The problem with the presentation given earlier is that we know of no simple full families of maps the homterval problem means that standard families, for example with branches of the form constant plus $x^{\delta}$ with $\delta>1$ which arise in homoclinic bifurcations are not full families $[9,12]$. Take for example the renormalization in $B_{1}$ : the induced map $R_{B_{1}} f_{\mathbf{a}}: I \rightarrow I$ defined by

$$
R_{B_{1}} f_{\mathbf{a}}(x)= \begin{cases}f_{\mathbf{a}}(x) & \text { if } x<0 \\ f_{\mathbf{a}}^{2}(x) & \text { if } x>0\end{cases}
$$


is a map in class B. Moreover, $f_{\mathbf{a}}^{2}\left(0_{+}\right) \leq f_{\mathbf{a}}\left(0_{-}\right)$, with equality if $f_{\mathbf{a}}\left(0_{+}\right)=0$, and in the limit of $f_{\mathbf{a}}\left(0_{+}\right)=z_{0}$ (i.e. $z_{1} \rightarrow 0$ ), $f_{\mathbf{a}}^{2}\left(0_{+}\right)=z_{0}$. Hence $R_{B_{1}} f_{\mathbf{a}}$ is in either $B_{1}, B_{2}$, $B_{3}, L_{1}$ or $L_{2}$.

Dropping the subscript a, the maps $F$ in regions $B_{1}$ up to $L_{2}$ are defined via the inequalities $F\left(c_{-}\right)>c, F\left(c_{-}\right) \geq F\left(c_{+}\right)$and $F^{2}\left(c_{-}\right)<c$. So, in terms of the symbolic description, their kneading invariants $\left(K_{+}, K_{-}\right)$satisfy one of the three possibilities described in Lemma ??, and $K_{-}=-1+1-1 \ldots$ The region $B_{1}$ itself has maps with $f\left(c_{-}\right)>c$ and $f\left(c_{+}\right)<c$ and $f^{2}\left(c_{-}\right)<c$ follows from the condition on $c_{+}$. Thus its kneading invariant $\left(k_{+}, k_{-}\right)$satisfies the same three sets of inequalities, but $k_{+}=+1-1 \ldots$ and $k_{-}=-1+1-1 \ldots$

The statements made in the description of renormalization in section 7 are that (a) applying the transformation $+1 \rightarrow+1-1$ and $-1 \rightarrow-1$ to any $\left(K_{+}, K_{-}\right)$gives a kneading invariant $\left(k_{+}, k_{-}\right)$of a map in $B_{1}$, and conversely, (b) every kneading invariant of a map in $B_{1}$ is either in the class $\left(K_{+}, K_{-}\right)$or has zero entropy and a finite set of periodic orbits (symbolically different), or is chaotic.

Let us prove these statements separately.

Proposition 10. Let $\left(K_{+}, K_{-}\right)$be the kneading invariant of a msdc map $F$ in class $B$ with $F\left(c_{-}\right)>c, F\left(c_{-}\right) \geq F\left(c_{+}\right)$and $F^{2}\left(c_{-}\right)<c$. Then the pair $\left(k_{+}, k_{-}\right)$obtained by applying the transformations

$$
+1 \rightarrow+1-1, \quad-1 \rightarrow-1
$$

is the kneading invariant of a map $f$ in $B_{1}$ (i.e. with $f\left(c_{-}\right)>c$ and $f\left(c_{+}\right)<c$ ).

Proof: First note that since (88) preserves parity, the map $f$ if it exists will be in class B. Also every +1 in $k_{ \pm}$is followed by a sequence $(-1)^{n}$ for some $1 \leq n \leq \infty$ consistent with $f \in B_{1}$. Since $F\left(c_{-}\right)>c$ and $F^{2}\left(c_{-}\right)<c, K_{-}=-1+1-1 \ldots$ and so $k_{-}=-1+1-1-1 \ldots$, i.e. $f\left(c_{-}\right)>c$ and $f^{2}\left(c_{-}\right)<c$.

We now need to consider the different possibilities for $\left(K_{+}, K_{-}\right)$as outlined in section 6 . The first case is given by (45) and (46), i.e.

$$
\text { either } \sigma^{2} K_{-} \prec_{s o+} \sigma^{r} K_{+} \prec_{s o+} K_{-} \text {or } K_{+} \preceq_{s o+} \sigma^{r} K_{+} \prec_{s o+} \sigma K_{-}
$$

depending on whether $\sigma^{r} K_{+}$starts with a minus one or plus one respectively, and

$$
\text { either } \sigma^{2} K_{-} \preceq_{s o+} \sigma^{r} K_{-} \preceq_{s o+} K_{-} \text {or } K_{+} \prec_{s o+} \sigma^{r} K_{-} \preceq_{s o+} \sigma K_{-}
$$

depending on whether $\sigma^{r} K_{-}$starts with a minus one or plus one respectively, where $r>0$ for $K_{+}$and $r>2$ for $K_{-}$. We want to show that the pair $\left(k_{+}, k_{-}\right)$obtained by applying (88) to $\left(K_{+}, K_{-}\right)$satisfies a similar set of inequalities (we have already shown in the first paragraph that if it is admissible, then the kneading invariant must be for a map in $B_{1}$ ).

Suppose that $\sigma^{r} k_{+}=+1 \ldots$ but that $\sigma k_{-} \preceq_{s o+} \sigma^{r} k_{+}$. Since $k_{+}$can be written in terms of factors of $(+1-1)$ and $(-1)$ we know that $\sigma^{r} k_{+}=(+1-1)^{u_{1}}(-1)^{u_{2}} \ldots$ 
where $\sigma^{s} K_{+}=(+1)^{u_{1}}(-1)^{u_{2}} \ldots$ Similarly, $\sigma k_{-}=(+1-1)^{a_{1}}(-1)^{a_{2}} \ldots$ where $\sigma K_{-}=$ $(+1)^{a_{1}}(-1)^{a_{2}} \ldots$ Thus if $\sigma k_{-}=\sigma^{r} k_{+}$we find $\sigma K_{-}=\sigma^{s} K_{+}$a contradiction. Now suppose $\sigma k_{-} \prec_{\text {so+ }} \sigma^{r} k_{+}$. Since the two sequences differ there is a word $w$ of length $q$ (say) such that $\sigma k_{-}=w A$ and $\sigma^{r} k_{+}=w B$, and the sequences $A$ and $B$ start with different symbols. If $w$ has an even number of plus ones then $\sigma k_{-} \prec_{s o+} \sigma^{r} k_{+}$ implies that $A=-1 \ldots$ and $B=+1 \ldots$ and if $w$ has an odd number of plus ones then $A=+1 \ldots$ and $B=-1 \ldots$ Note that $w$ cannot end with plus one, as every plus one is followed by a minus one, and so this could not be the point at which the sequences differ. Thus $w=(+1-1)^{a_{1}} \ldots(+1-1)^{a_{v-1}}(-1)^{a_{v}}$ (with $a_{v}=0$ possible) and

$$
\sigma K_{-}=(+1)^{a_{1}} \ldots(+1)^{a_{v-1}}(-1)^{a_{v}}(-1)^{a_{v+1}}(+1)^{a_{v+1}} \ldots
$$

If $w$ is even/or odd so is $W=(+1)^{a_{1}} \ldots(+1)^{a_{v-1}}(-1)^{a_{v}}$. Suppose $w$ is even, then $\sigma k_{-}=w(-1) \ldots$ and $\sigma^{r} k_{+}=w(+1) \ldots$ implies that $\sigma K_{-}=W(-1) \ldots$ and $\sigma^{s} K_{+}=$ $W(+1) \ldots$ and so $\sigma K_{-} \prec_{s o+} \sigma^{s} K_{+}$, another contradiction. If $w$ is odd a similar argument comes to the same conclusion.

Still with the case $\sigma^{r} k_{+}=+1 \ldots$, the assumption that $\sigma^{r} k_{+} \prec_{\text {so+ }} k_{+}$leads to a similar contradiction $\left(\sigma^{s} K_{+} \prec_{s o+} K_{+}\right.$, with $\left.\sigma^{s} K_{+}=+1 \ldots\right)$.

The other cases (i.e. (46) are similar (and in many cases can be reduced to those done above).

The second case for the kneading invariant $\left(K_{+}, K_{-}\right)$is where $\sigma^{p} K_{+}=K_{-}$or $\sigma^{p} K_{-}=K_{+}$are also similar, so we conclude with the third case: $K_{+}=\left(W_{+} W_{-}\right)^{\infty}$, $K_{-}=\left(W_{-} W_{+}\right)^{\infty}$ where $W_{-}$has length $Q$ and $W_{+}$has length $P$ with inequalities (49) and (50) holding. Suppose that $\left(w_{-} w_{+}\right)^{\infty} \prec_{s o+} \sigma^{k}\left(w_{-} w_{+}\right) \infty$, where $w_{ \pm}$are the words created by applying (88) to $W_{ \pm}$, and $k<|Q|$. If $\sigma^{k} W_{-}$breaks $W_{-}$at a point where it is 'in sync' with the factorization in terms of $(-1)$ and $(+1-1)$ then a simple argument leads to a contradiction for $K_{ \pm}$. Thus $w_{-}=(a(+1))(-1 b)$ where the length of $(a(+1))$ is $k$. and the hypothesis is that

$$
k_{-}=w_{-} w_{+} \ldots \prec_{s o+}(-1) b w_{+} w_{-} \ldots
$$

But of course, $w_{-}$starts with a -1 , so this implies that

$$
\sigma k_{-} \prec_{s o+} b w_{+} w_{-} \cdots
$$

and since $b$ is 'in sync' again, we obtain a contradiction.

We will not pursue the proof further - there are so many different cases that the understanding gained would be outweighed by the boredom attained. Indeed, the methods indicated above are sufficient to convince yourself of the following.

Proposition 11. If $f \in B_{1}$ has kneading invariant $\left(k_{+}, k_{-}\right)$then the sequences $K \pm$ obtained by applying the transformation

$$
+1-1 \rightarrow+1, \quad-1 \rightarrow-1
$$

are the kneading invariant of a map $F$ in class $B$ with $F\left(c_{-}\right)>F\left(c_{+}\right)$and $F\left(c_{-}\right)>c$. 
The renormalization for maps in $B_{1}$ is particularly simple in that they can always be renormalized; there is no stopping rule which restricts the next level of renormalization. In the other cases it is necessary to eliminate the positive entropy cases as indicated in section 7 .

\section{Conclusion And CAveats}

In this paper a subshift of finite type for the renormalization of two classes of single discontinuity maps related to differential equations has been given explicitly. This makes it possible to give a complete description of the periods present for maps on the boundary of chaos in these classes. Whilst this seems new and interesting a number of caveats are worth making (the reader may prefer to see these as assumptions).

1. The mathematics described works entirely on the symbolic level of kneading theory. We are not aware of any results which prove that full families of such maps exist, nor that the natural parametrization of such maps if they do exist can be mapped to the plane. In particular, it has not been proved that the quadratic examples of section two satisfy all the assumptions needed. In consequence, it may turn out that not all the (uncountably many) routes to chaos described by the subshift are realizable for differentiable maps in the relevant classes. On the other hand, given any sequence of renormalizations it is straight-forward to construct maps in class B or C which are renormalizable with this sequence of renormalizations (provided of course that the sequence is allowed by the subshift).

2. When talking about points and lines on the boundary of chaos we have been assuming metric properties of the subshift without justification. From [19] we know that there are lines in each A-box on which the transition to chaos is abrupt, via a mechanism related to circle intermittency. Similarly we know that in each B-box and C-box there are segments of lines on which the transition to chaos is via the anharmonic bifurcation [14]. On the other hand, it is not known whether there are any other robust routes to chaos: numerical evidence suggests that these are the only two. Hence we have assumed that all other routes correspond to point on the boundary of chaos.

3. Section five gives an algorithm for calculating the periods of periodic orbits for maps on the boundary of chaos. The representation does not always give monotonic sequences and there may well be a better way of describing the periods.

4. (Historical note) This paper is adapted from a preprint which formed a chapter of my Adams Prize Essay in 1992. It was not submitted for publication at the time because I could not prove that any given family is full (indeed, there are results to prove that standard families are not full $[8,9,12])$. However, the renewed interest in piecewise monotonic maps in hybrid systems, and the approach taken in section four 
which shows that every admissible sequence in the kneading invariant of a piecewise continuous map, makes me feel that this is worth publishing now.

AcknowledgementsThis research was partially funded by the CICADA project, EPSRC grant EP/E050441/1.

\section{REFERENCES}

[1] V. Avrutin and M. Schanz (2006) 'On multi-parametric bifurcations in a scalar piecewise-linear map', Nonlinearity $19531-552$.

[2] V. Avrutin and M. Schanz (2008) 'On the fully developed bandcount adding scenario', Nonlinearity 21 1077-1103.

[3] V. Avrutin, M. Schanz and S. Bannerjee (2006) 'Multi-parametric bifurcations in a piecewiselinear discontinuous map', Nonlinearity 19 1875-1906.

[4] S. Bannerjee, M.S. Karthik, G. Yuan and J.A. Yorke (2000) 'Bifurcations in one-dimensional piecewise smooth maps - theory and applications in switching circuits',

[5] M. di Bernardo, C.J. Budd and A.R. Champneys (1998) 'Grazing, skipping and sliding: analysis of the nonsmooth dynamics of the dc/dc buck converter', Nonlinearity 11 858-890.

[6] M. di Bernardo, C.J. Budd and A.R. Champneys (2001) 'Normal form maps for grazing bifurcations in $n$-dimensional piecewise-smooth dynamical systems', Physica D 160 222-254.

[7] M. di Bernardo, C.J. Budd, A.R. Champneys, P. Kowalczyk (2008) Piecewise-smooth Dynamical Systems: Theory and Applications, Springer, London.

[8] D. Berry (1990) 'Nonwandering sets of Lorenz maps' Ph.D. thesis, University of Warwick.

[9] D. Berry and B.D Mestel (1991) 'Wandering Intervals for Lorenz maps with Bounded Nonlinerity', Bull. LMS 23 183-189.

[10] P. Collet and J.-P. Eckmann (1981) Iterated maps on the interval as dynamical systems, Birkhäuser, Basel.

[11] J.M. Gambaudo, I. Procaccia, S. Thomae and C. Tresser (1987) 'New universal scenarios for the onset of chaos in Lorenz-type flows' Phys. Rev. Lett. 57 925-928.

[12] P. Glendinning (1992) Chaos and Routes to Chaos in Lorenz maps, Adams Prize Essay, University of Cambridge.

[13] P. Glendinning (1992) 'Robust new routes to chaos in differential equations', Phys. Lett. A 168 40-46.

[14] P. Glendinning (1993) 'The anharmonic route to chaos: kneading theory', Nonlinearity 6 349367.

[15] P. Glendinning, J.E. Los and C. Tresser (1990) 'Renormalization between classes of maps' Phys. Lett. A 145 109-122.

[16] J.H. Hubbard and C.T. Sparrow (1990) 'The Classification of Topologically Expansive Lorenz Maps', Comm. Pure Appl. Math., XLIII 431-443.

[17] D.V. Lyubimov, A.S. Pikovsky and M.A. Zaks (1989) 'Universal scenarios of transition to chaos via homoclinic bifurcations', Sov. Sci. Rev. C. Math. Phys. 8 221-292.

[18] Milnor and Thurston

[19] C. Tresser (1983) 'Nouveaux types de transitions vers une entropie topologique positive' C.R.Acad. Sci. (Paris) Série I, 296, 729-732. 